\title{
Electron spin teleportation current through a quantum dot array operating in the stationary regime
}

\author{
Olivier Sauret ${ }^{a}$, Denis Feinberg ${ }^{a}$, Thierry Martin $^{b}$ \\ ${ }^{a}$ Laboratoire d'Etudes des Propriétes Electroniques des Solides, Centre National de la Recherche Scientifique, BP166, 38042 \\ Grenoble, France \\ ${ }^{b}$ Centre de Physique Théorique et Université de la Méditerranée, Case 907, 13288 Marseille, France
}

\begin{abstract}
An electron spin state teleportation scheme is described in detail. It is based on the protocol by Bennett et al. [Phys. Rev. Lett. 70, 1895 (1993)], and involves the production and detection by superconductors of entangled pairs of electrons. Quantum dots filter individual electron transitions, and the whole teleportation sequence is selected in a five-dot cell by electrostatic gating in the stationary regime (no time dependent gate voltages): i) a normal dot carry the electron spin state to be teleported, two others carry the ancillary entangled pair; ii) two superconducting dots, coupled by a superconducting circuit, control the injection of the source electron and the detection of the teleported electron. This teleportation cell is coupled to emitter and receiver reservoirs. In a steady state, a spin-conserving current flows between the reservoirs, most exclusively carried by the teleportation channel. This current is perfectly correlated to a Cooper pair current flowing in the superconducting circuit, and which triggers detection of the teleported electron. This latter current indeed carries the classical information, which is necessary to achieve teleportation. The average teleportation current is calculated using the Bloch equations, for weakly coupled spin reservoirs. A diagnosis of teleportation is proposed using noise correlations.
\end{abstract}

PACS 74.50+r,73.23.Hk,03.65.Ud

\section{INTRODUCTION}

Teleportation belongs to fundamental science since Bennett and coworkers proposed a protocol for quantum teleportation of a two-state particle [1]. This means reconstructing the quantum state of a particle at a distant place, on a preexisting particle which state was previously undetermined. Of course, any measurement of the quantum state to be teleported must be avoided whatsoever. Bennett et al. proposed to take advantage of the non-locality of quantum mechanics [2,3], celebrated in the EPR "paradox" [4. To this purpose, a pair of entangled particles is produced. This means that the state of each of them is undetermined, while it is fully determined once a measurement is made on the other. One member of the pair is given to the sender, Alice, the other one (the target) to the receiver, Bob. Alice also receives a "source" particle in an unknown state, which she wants to teleport to Bob. Then she performs a joint measurement on this particle and her member of the entangled pair, so that as to measure them in an entangled state. As a result, the state of the target member of the pair, in the receiver (Bob)'s hands, is simultaneously determined. Alice must send the result of her measurement as a classical signal. The state of the source particle can then be retrieved by Bob, by applying to the target particle a unitary transformation. The state of the source particle has been destroyed during the process (no-cloning theorem [5]). An essential point is that, despite the simultaneity of Alice's measurement and Bob's particle state projection, teleportation is completed only when the classical information about the result of the joint measurement (four possible Bell states thus two classical bits) has been received. Therefore, as a mean of transmitting information, TP does not violate any fundamental law. The quantum information stored in the original state (qubit) has been split into a "quantum" channel (the entangled pair) and a classical channel.

The first experimental verification of teleportation has been performed with photons [6], and was followed by several others [7]. Entangled photons with correlated orthogonal polarizations (antisymmetrical state) were produced by parametric-down conversion, and measurement of one of the four possible entangled states (the singlet) was achieved by polarized beam splitters. This simplification changes a little, though not fundamentally, the scenario of Bennett et al.: the classical signal carries simply a "yes" or "no" answer concerning detection, and if the answer is yes, Bob has readily in hands the original state, without needing any further transformation. As shown by the authors of Ref. [6], this simplification does not affect the quantum correlations of the input and output particles (defined as the fidelity of TP), but it reduces the efficiency of TP (success has a probability 1/4). The TP protocol is thus rendered slower by a factor $1 / 4$. In practice, unambiguous detection of teleportation requires coincidence measurements of photons at four detectors (one for the detected particle, two for the Bell measurement and one for a test for emission of the source particle), and it relies on the optimal control of individual photons achieved in modern quantum optics devices. It is in fact important to keep track of the emitted pair and the source particle, in order to control that Bob indeed 
measures the twin of the photon which experiences the joint measurement by Alice, and not a member of a previously or a subsequent emitted pair (in which case no correlation would be expected).

The proposal of Bennett and coworkers and the subsequent experiments in quantum optics or atomic physics [6, []] and NMR [8] provide a beautiful illustration of the power of entanglement as a basic resource for quantum information [9]. TP appears as a promising way to send unaltered quantum information, for instance by entangling fragile qubits with more robust ones, or with qubits that can be propagated over long distances (photons for instance). The same principle allows to swap entanglement between successive particles, therefore entangling distant ones. Other applications include distributing information among networks, or error-correcting codes [10].

Search for scalability naturally leads to a quest for similar processes in solid-state environments. The idea of electron/spin transport for quantum information processing schemes has also been developed in Refs. 11]. Here we shall focus on electron transfer between dots. In fact the strong advantages of photons (weak interactions with the environment, allowing long-distance coherent propagation) turn out to be also inconveniences : single or pair photon sources are weak, and it is difficult to operate gates on photon ensembles since they interact weakly, only through non-linear media. On the other hand, electrons can be produced one by one, using Coulomb blockade in quantum dots, or in pairs 12, 13, 14, 15.

Since electrons are charged particles, it is in principle possible to operate with a variety of gates on them, taking advantage of their Coulomb interactions in nanostructures. Electronic systems also have obvious advantages toward integration. The main drawback of electronic proposals is that the underlying interactions can also lead to strong decoherence effects. Yet, because high intensity single electron sources can be operated, the relevant time scales can be very short, and there is hope that quantum coherence of individual qubits can be controlled over distances ranging between microns and millimeters.

Exploring entanglement in the solid state concerns the practical manipulation of qubits, but also the investigation of fundamental phenomena such as a proof of non-locality with massive or fermionic particles like electrons [16]. An existing proposal for TP considers excitons in coupled quantum boxes, that can be manipulated optically [17]. Alternatively, an important issue arises when considering the electron spin degree of freedom, which is a candidate as a qubit for integrated quantum information devices [18]. To produce electron pairs in an entangled spin state, one needs as a source a device where electron spins are correlated by their previous interactions or by their statistics, but the two particles can be dissociated while keeping this correlation. Up to now, proposals have been made using i) Cooper pairs in superconductors [15, 12, 13, 14] or ii) singlet states on a discrete level in quantum dots [19,20]. Entangled pairs can then be produced by the use of energy [12,13, 14, 19, 20] or spin filtering [15, 14]. Starting from this elementary unit for entanglement, the construction of more complex devices relies on the analogy between photon propagation in waveguides and phase-coherent electron transport in nanostructures, which have been well illustrated by the fermion version of the Hanbury-Brown and Twiss intensity correlations [21,22]. However, further possibilities are opened by using Coulomb interactions : transport of electrons, one electron at a time, can indeed be forced with the help of electrostatic barriers. Electrons are trapped in quantum dots, separated by tunnel barriers and their transitions between dots and reservoirs are controlled by electrostatic gates [23]. Moreover, as shown in this work, correlations between transitions of different electrons at different barriers can indeed be used as a powerful tool to control and manipulate the spin qubits. Then, although time-resolved control of individual electrons is still out of reach, teleportation can be achieved in a steady state operation through electrostatic couplings only [24]. For a correct choice of the system parameters, we show in the following that an elaborate sequence of transitions can be selected. For instance, in the teleportation process, a strict control is required on the time sequence: the joint measurement of the source particle (1) and of the particle (2) must occur only after the pair (2,3) is emitted, and the detection of the "teleported" particle (3) must wait for the joint measurement of particles (1,2). As explained in detail in the present paper, electrostatic couplings between dots, together with individual gating, provide the necessary correlations to filter a unique transition sequence through the cell, without any temporal gate control whatsoever.

In the following, a detailed description of the operation of the teleportation cell is presented. An overall qualitative presentation is the subject of Section II. A full derivation of the microscopic and effective Hamiltonians is given in Section III, and justifications are provided in Appendix A. Section IV contains the discussion of the electrostatics of the cell and the selection of the teleportation sequence. Section V is devoted to the calculation of the steady state teleportation current using a Bloch equation approach. Section VI discusses the overall similarities and differences with the quantum optics implementation of teleportation. A summary of our results, also discussing possible extensions of our solid state teleportation proposal, is provided in the conclusion (Section VII). 


\section{QUALITATIVE DESCRIPTION OF THE TELEPORTATION PROTOCOL}

\section{A. Description of the teleportation cell}

Let us describe the set-up which allows teleportation of the electron spin. The TP cell is defined as comprising the dots $\mathbf{1}, \mathbf{2}, \mathbf{3}, \mathbf{a}, \mathbf{b}$ (see Fig. 1) and the circuit $S$ (Fig. 2a). The basic resource, the entangled pair of electrons, is produced by superconducting dot $\mathbf{b}$ according to the recent proposal 12.13]: two normal quantum dots (numbered $\mathbf{2}$ and 3) form neighboring tunnel junctions with a singlet superconducting electrode. Electrostatic gates tune the dot chemical potentials such that the transition of a Cooper pair from the superconductor to the couple of dots is resonant if and only if each partner of the pair is sent to a different dot : conversely, adding to or subtracting two electrons from a single dot is strongly suppressed by Coulomb energy. Due to the symmetry of the Cooper pair wave function in the superconductor, the two electrons which are added in dots $\mathbf{2 , 3}$ are in the antisymmetric singlet state, which is entangled. Among the four Bell states available with two spin $\frac{1}{2}$ particle, none of the three triplet combinations can be created. The double tunneling process involved in the creation of the entangled state is often denoted as a non-local (or crossed) Andreev process [15,25]. Its amplitude depends on the tunneling amplitudes at both junctions (assumed equal for simplicity), and on the distance $l$ between junctions [12,25,26]. The latter dependence involves exponential decay of quasiparticles in the bulk (clean) superconductor (on the coherence length $\xi_{0}$ ) and an algebraic factor $\left(\lambda_{F} / l\right)^{2}$. Therefore a basic limitation of the "Andreev entangler" is that the distance between the two junctions must not typically exceed a few nanometers. Improvement of the algebraic factor is obtained by reducing the effective dimensionality of the superconductor [27,28] or using a "dirty" superconductor [29].

The source electron is created as an additional charge in dot 1 . The analyzer, which allows "Alice" to perform the joint measurement, is chosen to be another superconductor. This possibility of using the same physical phenomenon both for production and detection of entangled pairs, is specific to solid state nano-electronics. This is in contrast with optics where parametric down conversion produce the pairs and polarized beam splitters detect antisymmetric pairs in an irreversible way. In the present case, having an irreversible transition of two electrons tunneling from dots 1 and $\mathbf{2}$ to superconductor a (Alice) provides a measurement of those electrons in a singlet state. Note that if the electrons were to oscillate between dots $\mathbf{1}$ and $\mathbf{2}$ (as a singlet) and dot a (as a Cooper pair) there would be no measurement whatsoever. Below, we shall see that the irreversibility is brough the presence of a reservoir which injects an electron in 1. This is analogous to the measurement performed by Alice in the Innsbruck optics experiment [6]. In this experiment, the two photons which form the singlet state are destroyed by the measurement process.

If needed, the production of a spin-polarized electron can be achieved by a spin-polarized reservoir $\mathbf{L}$, connected to dot 1 by a tunnel junction. This polarized source could be made of a strongly polarized ferromagnet (half-metal) or based on any other injection scheme, using semiconductors for instance. Symmetrically, detection of the teleported electron, sitting in dot $\mathbf{3}$, can be achieved by a spin-polarized reservoir $\mathbf{R}$, in the hands of "Bob" (Figure 1).

It is convenient to connect the superconducting electrodes $\mathbf{a}$ and $\mathbf{b}$ by a superconducting circuit $\mathbf{S}$. Indeed, once Alice in a detects a singlet pair, this pair can flow through the circuit $\mathbf{S}$ towards $\mathbf{b}$, where, as we shall see, it triggers detection by Bob. In addition, optimum operation of the device requires to correlate the charge transitions in the normal dots and superconducting electrodes. To this purpose, $\mathbf{a}$ and $\mathbf{b}$ are chosen to be superconducting dots with sizeable Coulomb charging energy. This enables to absorb/eject electron pairs one by one.

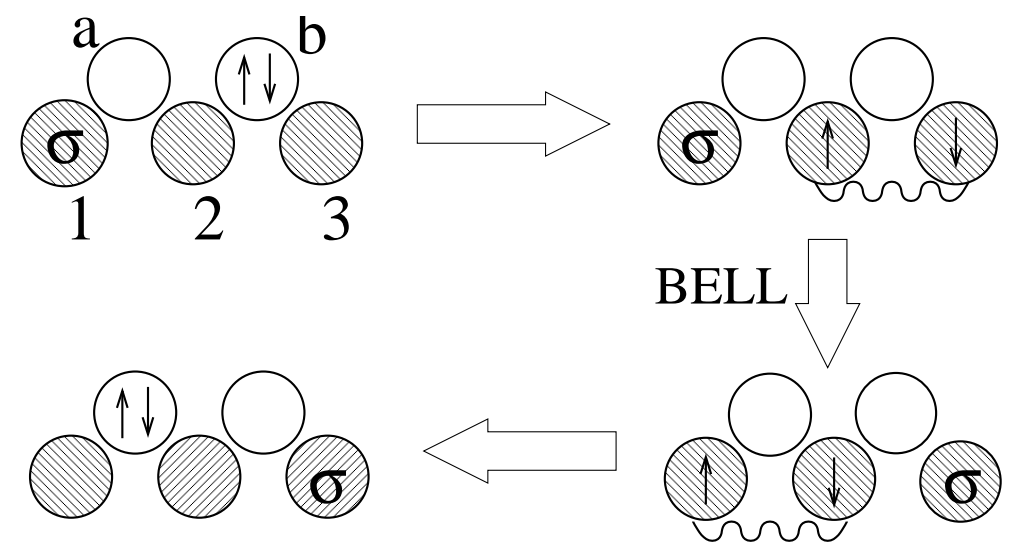


FIG. 1. The elementary teleportation sequence. 3 normal dots (shaded) labelled $\mathbf{1 , 2}, \mathbf{3}$ can only accommodate 0 or 1 electron, while the 2 superconducting dots $\mathbf{a}, \mathbf{b}$ can accommodate 0 or two electrons in a Cooper pair state. The sequence starts with an injection process from reservoir $L$, followed by the Bell decomposition of the 3 electron wave function in the normal dots, and terminates by the detection in $R$.

\section{B. Bell state decomposition}

Before discussing the conditions on the parameters of this set-up, let us recall why the measurement on $(\mathbf{1}, \mathbf{2})$ achieves teleportation [1, 6]. Having an electron in dot $\mathbf{1}$ in the state:

$$
|\sigma\rangle_{1}=\alpha|\uparrow\rangle_{1}+\beta|\downarrow\rangle_{1}
$$

an entangled pair of electrons is created in dots 2,3 in the singlet state $\left|\Psi^{S}\right\rangle_{23}$. The latter is one of the four basis orthonormal Bell states (one singlet and three triplets)

$$
\begin{aligned}
\left|\Psi^{S}\right\rangle & =2^{-1 / 2}(|\uparrow \downarrow\rangle-|\downarrow \uparrow\rangle) ; & & \left|\Psi^{T 0}\right\rangle=2^{-1 / 2}(|\uparrow \downarrow\rangle+|\downarrow \uparrow\rangle) \\
\left|\Psi^{T-}\right\rangle & =2^{-1 / 2}(|\uparrow \uparrow\rangle-|\downarrow \downarrow\rangle) ; & & \left|\Psi^{T+}\right\rangle=2^{-1 / 2}(|\uparrow \uparrow\rangle+|\downarrow \downarrow\rangle)
\end{aligned}
$$

One verifies that the resulting three-electron state can be rewritten as

$$
\begin{aligned}
|\Psi\rangle_{123}=|\sigma\rangle_{1}\left|\Psi^{S}\right\rangle_{23}= & -\frac{1}{2}\left|\Psi^{S}\right\rangle_{12}\left(\alpha|\uparrow\rangle_{3}+\beta|\downarrow\rangle_{3}\right)+\frac{1}{2}\left|\Psi^{T 0}\right\rangle_{12}\left(-\alpha|\uparrow\rangle_{3}+\beta|\downarrow\rangle_{3}\right) \\
& +\frac{1}{2}\left|\Psi^{T-}\right\rangle_{12}\left(\beta|\uparrow\rangle_{3}+\alpha|\downarrow\rangle_{3}\right)+\frac{1}{2}\left|\Psi^{T+}\right\rangle_{12}\left(-\beta|\uparrow\rangle_{3}+\alpha|\downarrow\rangle_{3}\right)
\end{aligned}
$$

In the original scenario [1], Alice is able to perform a measurement of any Bell state on particles $\mathbf{1}, \mathbf{2}$. This at the same time projects the state of particle $\mathbf{3}$ onto a particular state, which Bob can transform into the original state $|\Psi\rangle_{3}=|\sigma\rangle_{3}=\alpha|\uparrow\rangle_{3}+\beta|\downarrow\rangle_{3}$ by applying an appropriate unitary transformation. The latter is known when Alice sends by a classical two-bit channel the result of her measurement, which therefore completes teleportation. In particular, when the result is the singlet $\left|\Psi^{S}\right\rangle$, the state in hands of Bob is nothing but the original state of particle 1, up to a minus sign. Bouwmester et al. [6,30] simplified this protocol : measuring only the state $\left|\Psi^{S}\right\rangle$ still allows teleportation, provided Alice sends as an information that she indeed completed the measurement.

The set-up we propose for teleportation of electron spins is similar in its principle. Once three additional electrons are in dots $\mathbf{1}, \mathbf{2}, \mathbf{3}$, the three possible triplet states for electrons in 1, $\mathbf{2}$ lead to no detection, while the singlet state can trigger (with probability $\frac{1}{4}$ ) an Andreev transition to the superconducting dot a, which acts as Alice's measurement apparatus. The remaining spin in dot $\mathbf{3}$ (target) acquires the same state $\sigma$ as the initial spin in dot 1, but teleportation is completed only when the electron is detected in reservoir $\mathbf{R}$ (Bob).

\section{The teleportation sequence}

The teleportation sequence (see Fig. 1) is the following : having an additional electron in dot 1, an entangled pair is created in dots $(\mathbf{2}, \mathbf{3})$. Thus one electron occupies each of the normal dots. If the two electrons in $(\mathbf{1}, \mathbf{2})$ are in the singlet state, they can tunnel in dot a. This becomes a true measurement when another electron enters dot $\mathbf{1}$ from lead $\mathbf{L}$, blocking the singlet pair in dot a. At the same time the spin state of the electron previously in $\mathbf{1}$ is transfered to 3, as shown by Eq. 3. Let us show how this teleportation sequence is embedded in a teleportation cycle. Indeed (see Fig. 4), the pair absorbed in a can flow from $\mathbf{a}$ to $\mathbf{b}$ and trigger the transfer and detection of the teleported electron in $\mathbf{R}$, thus allowing to recover the initial state in Fig. 1. It can then serve as source for another entangled pair, for the next teleportation cycle (Fig. A). It is essential to notice that irreversibility of the pair production (from $\mathbf{b}$ ) and of the measurement (in $\mathbf{a}$ ) is provided by the coupling to the reservoirs : Coulomb repulsion between dots $\mathbf{b}$ and $\mathbf{3}$ makes the "target" electron in $\mathbf{3}$ leave towards reservoir $\mathbf{R}$, which in turn lowers the energy of the pair in dot $\mathbf{b}$, allowing resonance with $(\mathbf{2}, \mathbf{3})$. Similarly, the pair in $(\mathbf{1}, \mathbf{2})$ oscillates back and forth to a, but it becomes localized in $\mathbf{a}$ when a new source electron enters dot $\mathbf{1}$. This in turn raises the energy in dot $\mathbf{a}$ and allows resonance between $\mathbf{a}$ and $\mathbf{b}$. The above set-up, together with static gating of dots $\mathbf{1}, \mathbf{2}, \mathbf{3}$, allows efficient filtering of single-electron transitions (from $\mathbf{L}$ to $\mathbf{1}$ and $\mathbf{3}$ to $\mathbf{R}$ ) and two-electron transitions (from $\mathbf{b}$ to $(\mathbf{2}, \mathbf{3}$ ) and from $(\mathbf{1}, \mathbf{2})$ to $\mathbf{a}$ ). But it should also prevent spurious transitions : it is indeed essential, to ensure fidelity of the teleportation process, that the target electron in $\mathbf{3}$ does not escape (is detected by Bob) before Alice performs the measurement in a. As shown in detail in Section 
IV, this is obtained by appropriately choosing the gate potentials of each dots. The Coulomb correlations induced by the electrostatics of this five-dot system can indeed exactly select the correct teleportation sequence.

Let us emphasize that transfer of a charge $2 e$ from dot $\mathbf{a}$ to dot $\mathbf{b}$ plays the role of the classical channel. Even more, this classical signal strictly conditions the exit of the target electron. Therefore, according to the laws of quantum mechanics, teleportation is achieved without ambiguity, with a fidelity conditioned by the few spurious processes like cotunneling. This is in contrast with the optics experiment where (externally operated) time correlations are used to select the TP events from spurious ones.

The teleportation process manifests itself as a spin-conserving current passing from reservoir $\mathbf{L}$ to reservoir $\mathbf{R}$. Below we shall consider a situation where the cell (excluding the leads) is chosen to be symmetric, an assumption which allows to reduce the number of parameters. Then, the direction of this current is fixed by applying a voltage bias $V$ between $\mathbf{L} / \mathbf{1}$ and $\mathbf{3} / \mathbf{R}$. For each electron disappearing in $\mathbf{L}$ and each electron appearing in $\mathbf{R}$ with the same spin, exactly one Cooper pair flows through the circuit $\mathbf{S}$. Yet, in the present electronic device, a full proof of TP irrespective of a quantum or classical description - requires an additional diagnosis, which we discuss at the end of the paper.

\section{MODEL}

\section{A. Microscopic Hamiltonian}

The system under consideration (TP cell and reservoirs) is described by the Hamiltonian:

$$
H=H_{0}+H_{C}+H_{T}
$$

where $H_{0}$ contains the energy levels of the isolated elements (dots and reservoirs), $H_{C}$ is the total Coulomb charging energy and $H_{T}$ is a tunneling Hamiltonian. The part $H_{0}$ reads

$$
H_{0}=H_{1}+H_{2}+H_{3}+H_{a}+H_{b}+H_{S}+H_{L}+H_{R}
$$

with one-electron energy levels $\varepsilon_{i l}$ in the normal dots (in the Hartree approximation) $(i=1,2,3)$

$$
H_{i}=\sum_{l \sigma} \varepsilon_{i l} c_{i l \sigma}^{\dagger} c_{i l \sigma}
$$

Normal dots 1, 2, 3 must necessarily have a discrete spectrum, in order to avoid spin exchange processes which destroy entanglement. The "empty" state corresponds to a state with $2 N$ electrons in the dots, while the "singly occupied" state has an odd occupation number, which means the addition of one outer electron to the $2 N$ electrons within the dot 13]. The last doubly occupied level of dots $\mathbf{1}$ and $\mathbf{3}$ must lie well below the electro-chemical potential of reservoirs $\mathbf{L}$ and $\mathbf{R}$, so as to prohibit any spurious transition of "wrong" spin electrons between such levels and the reservoirs. This sets a lower bound on the level spacing [13].

Quasiparticle levels $E_{\alpha k}$ in the superconducting dots and reservoir $S(\alpha=a, b, S)$ enter the Hamiltonian for the superconductors:

$$
H_{\alpha}=\sum_{k \sigma} E_{\alpha k} \gamma_{\alpha k \sigma}^{\dagger} \gamma_{\alpha k \sigma}
$$

while in normal reservoirs $N=L, R$

$$
H_{N}=\sum_{k \sigma} \varepsilon_{N k \sigma} c_{N k \sigma}^{\dagger} c_{N k \sigma}
$$

In the above, the operators $c$ and $c^{\dagger}$ stand for electron annihilation and creation, and the $\gamma_{k \sigma}$ 's are the usual Bogolubov quasiparticle operators [31] $(\sigma= \pm 1), \gamma_{k \alpha \sigma}=u_{k \alpha} c_{k \alpha \sigma}+\sigma v_{k \alpha} c_{-k \alpha-\sigma}^{\dagger}$, with $u_{k \alpha}=(1 / \sqrt{2})\left(1+\xi_{k \alpha} / E_{k \alpha}\right)^{1 / 2}$, $v_{k}=(1 / \sqrt{2})\left(1-\xi_{k \alpha} / E_{k \alpha}\right)^{1 / 2}, \xi_{k \alpha}=\varepsilon_{k \alpha}-\mu_{\alpha}$ and $E_{\alpha k}=\sqrt{\xi_{k \alpha}^{2}+\Delta_{\alpha}^{2}}$. Here $\Delta_{\alpha}$ is the gap in superconductors $\alpha=\mathbf{a}, \mathbf{b}$, and $\mathbf{S}$ where the chemical potential is set to zero with respect to the normal reservoirs $\mathbf{L}, \mathbf{R}$.

One assumes a free-electron spectrum in reservoirs $L, R$ with spin-dependent quasiparticle energies. To a good approximation, a continuous BCS-like quasiparticle spectrum in reservoir $S$ and $\operatorname{dots} a, b$ is specified: this requires that the superconducting dots are "large" enough. Finally a discrete (and spin-independent) spectrum is chosen in $\operatorname{dots} 1,2,3$. 
The charging Hamiltonian can be written in a compact form as a function of the occupation numbers $N_{\mu}=$ $\sum_{\mu l} c_{\mu l \sigma}^{\dagger} c_{\mu l \sigma}$ of the dots $(\mu, \nu=1,2,3, a, b)$ [23, 32

$$
H_{C}=(1 / 2) \sum_{\mu, \nu} C_{\mu \nu}^{-1}\left(N_{\mu} e-Q_{\mu}\right)\left(N_{\nu} e-Q_{\nu}\right)
$$

where the $Q_{\mu}$ 's are the effective occupation number imposed by the gate and bias voltages (see Section IV and Appendix A). The couplings $C_{\mu \nu}^{-1}$ are the inverse capacitance matrix elements of the system.

Here the Coulomb charging energy of the superconducting dots is chosen to be smaller than their superconducting gap $e^{2} / C_{a, b} \ll \Delta_{a, b}$, so as to prohibit single electron transitions.

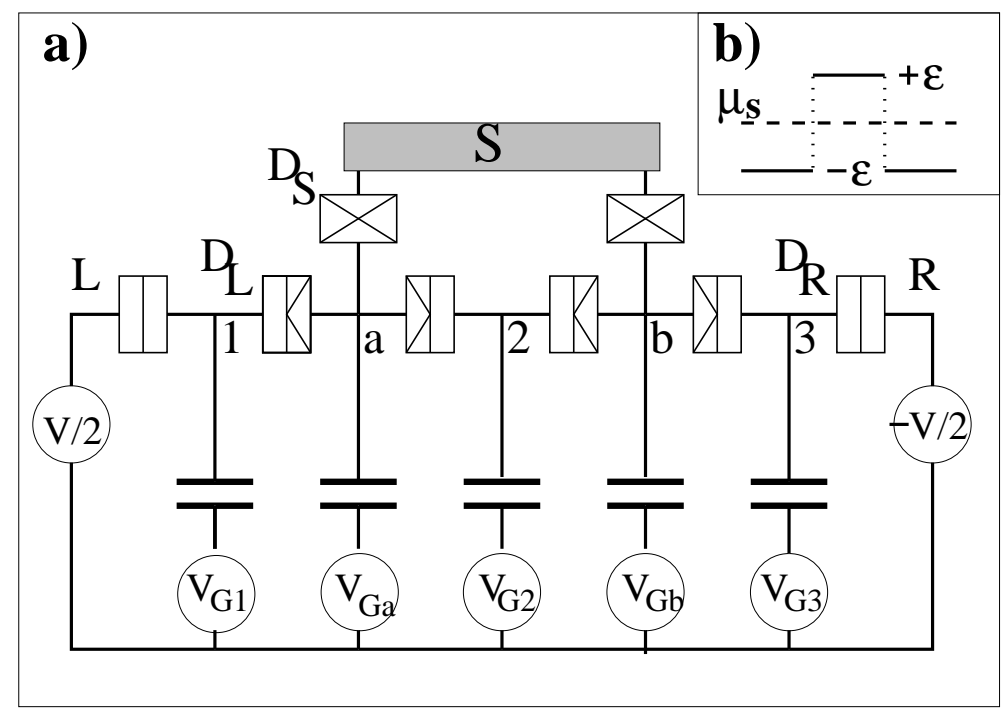

FIG. 2. a) The TP cell contains: i) NN junctions between reservoirs $\mathbf{L}, \mathbf{R}$ and dots $\mathbf{1}$ and $\mathbf{3}$; ii) N-S junctions between (1, a), $(\mathbf{a}, \mathbf{2}),(\mathbf{2}, \mathbf{b})$ and $(\mathbf{b}, \mathbf{3})$, and S-S junctions between a $(\mathbf{b})$ and the bulk superconductor $\mathcal{S}$. Detectors $D_{L, R, S}$ signal the passage of an electron/Cooper pair in the normal/superconducting circuit. b) Effective energy level configuration (from left to right) of dots $\mathbf{1}, \mathbf{2}$ and $\mathbf{3}$ ( $\mu_{S}$ is the superconductor chemical potential ).

Let us assume that electronic transitions involve only one level of the normal dots. This is justified at low enough temperature by the discrete spectrum of $\mathrm{N}$-dots, and by the fact that gate voltages can be chosen so as to select only two possible charge states. Tunneling is supposed to occur only between : reservoir/normal dot junctions $\mathbf{L} / \mathbf{1}, \mathbf{R} / \mathbf{3}$, normal dot/superconducting dot junctions $\mathbf{1} / \mathbf{a}, \mathbf{2} / \mathbf{a}, \mathbf{2} / \mathbf{b}, \mathbf{3} / \mathbf{b}$, and superconducting reservoir/superconducting dot junctions $\mathbf{S} / \mathbf{a}, \mathbf{S} / \mathbf{b}$. The one-electron tunneling Hamiltonian can then be written as

$$
H_{T}=H_{L 1}+H_{R 3}+H_{S a}+H_{S b}+H_{a 1}+H_{a 2}+H_{b 2}+H_{b 3}
$$

with

$$
\begin{aligned}
H_{T}= & \sum_{k \sigma} t_{L k} c_{L k \sigma}^{\dagger} c_{1 \sigma}+\sum_{k \sigma} t_{R k} c_{R k \sigma}^{\dagger} c_{3 \sigma}+\sum_{k k^{\prime} \sigma} t_{S a k k^{\prime}} c_{S k \sigma}^{\dagger} c_{a k^{\prime} \sigma}+\sum_{k k^{\prime} \sigma} t_{S b k k^{\prime}} c_{S k \sigma}^{\dagger} c_{b k^{\prime} \sigma} \\
& +\sum_{k \sigma} t_{a 1 k} c_{a k \sigma}^{\dagger} c_{1 \sigma}+\sum_{k \sigma} t_{a 2 k} c_{a k \sigma}^{\dagger} c_{2 \sigma}+\sum_{k \sigma} t_{b 2 k} c_{b k \sigma}^{\dagger} c_{2 \sigma}+\sum_{k \sigma} t_{b 3 k} c_{b k \sigma}^{\dagger} c_{3 \sigma}+\text { H.c. }
\end{aligned}
$$

Notice that no direct tunneling occurs between normal dots, instead, dot $\mathbf{2}$ is connected to both $\mathbf{a}$ and $\mathbf{b}$ dots. Tunneling through the junctions is controlled by the rates $\Gamma_{L(R) \sigma}=2 \pi t_{L 1(R 3)}^{2} \nu_{L(R) \sigma}(0), \Gamma_{S \alpha}=2 \pi t_{S \alpha}^{2} \nu_{S}(0)=\Gamma_{S}$ $(\alpha=a, b)$ and $\Gamma_{\alpha i}=2 \pi t_{\alpha i}^{2} \nu_{S}(0)=\Gamma_{\alpha}$ where $\nu_{L(R) \sigma}(0)$ is the spin-dependent density of states at the Fermi level in reservoirs $\mathbf{L}, \mathbf{R}$ and $\nu_{S}(0)$ the normal density of states in the superconducting reservoir.

An important constraint concerns the coupling between normal dots and the reservoirs. In order to avoid spin exchange between dots $\mathbf{1 , 3}$ and reservoirs, $\Gamma_{L}\left(\Gamma_{R}\right)$ must be smaller than the superconducting gap, the charging energy and the chemical potentials $\mu_{L}, \mu_{R}$ [13]. Moreover, the level spacings in each normal dot must be larger than $\mu_{L}, \mu_{R}$ (measured with respect to $\mu_{S}=0$ ). 


\section{B. Transport processes in the teleportation cell}

The microscopic Hamiltonian of the preceding section allows for several collective electron transfer processes. Some of these will be more favorable because of the intermediate states which they involve (generation of one or more quasiparticles in the superconducting elements). In particular, we will be interested in processes where a minimum of quasiparticles are excited in the superconducting elements of the teleportation cell.

First consider processes involving two electrons, being transfered from/to normal quantum dots and a superconducting dot. These are depicted in Fig. 3. Crossed Andreev reflection [12,25 - also called pair tunneling - (Fig. 3a) involves the quasi-simultaneous tunneling of two electrons to/from a superconducting dot to two neighboring dots. As the first electron tunnels it generates a quasiparticle, which is then destroyed by the second electron which is transfered. Cotunneling [33] describes the transfer of an electron from one normal quantum dot to another (Fig. 3b), via the superconductor (the order of the sequence of the two tunneling events is arbitrary). The intermediate state also implies the creation of a quasiparticle, which is subsequently destroyed as in pair tunneling, and cotunneling is a spin preserving transition.

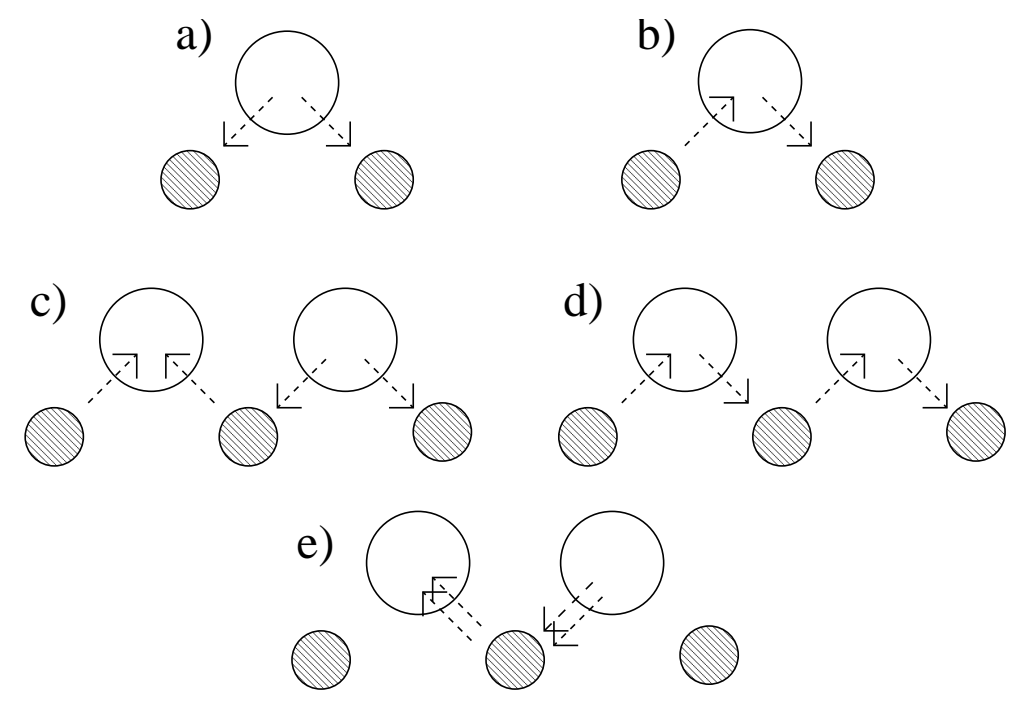

FIG. 3. Transport processes between normal quantum dots and superconducting dots: a) Crossed Andreev reflection; b) cotunneling; c) teleportation; d) sequence of two cotunneling events; e) Josephson tunneling through the central dot.

Next, there are three types of processes which involve 4 single electron jumps. Teleportation (Fig. 3c) requires that dot $\mathbf{1}$ is occupied initially, and dot $\mathbf{3}$ is occupied in the final state. A Cooper pair escapes from $\mathbf{b}$ and another one is absorbed in a. At no step in the process a directed matter transfer occurs between dot $\mathbf{1}$ and dot $\mathbf{3}$. This is the opposite case for the succession of two cotunneling events (Fig. 3 d d), where, conversely, no Cooper pair transfer occurs simultaneously, but an electron is transfered for $\mathbf{1}$ to $\mathbf{3}$ nevertheless.

Note that another possible cotunneling event from 1 to $\mathbf{3}$ can involve the bulk superconductor instead, by successive quasiparticle excitations in $\mathrm{S}$-dot $\mathbf{a}, \mathcal{S}$, and $\mathrm{S}$-dot b. However, this process is negligible: it involves a geometrical factor corresponding to the propagation of unpaired electrons between $\mathbf{a}$ and $\mathbf{b}$ through the bulk superconductor.

One may also consider the transfer of a Cooper pair from $\mathbf{b}$ to $\mathbf{a}$, this time without the transfer of an electron from 1 to 3 , which can be viewed as a kind of Josephson coupling between $\mathbf{b}$ and a [12, 34]. Note that both the successive cotunneling and the Josephson-like processes compete with the Andreev processes which are essential for teleportation.

Here, we argue that optimum conditions for the operation of the teleportation cell are met when the Andreev pair transitions (from $\mathbf{b}$ to $(\mathbf{2}, \mathbf{3})$ and from $(\mathbf{1}, \mathbf{2})$ to $\mathbf{a}$ ) are chosen to be resonant. The resonance condition also applies for the Cooper pair transitions between $\mathbf{a}, \mathbf{b}$ and the circuit $\mathbf{S}$. This Cooper pair transfer amplitude involves two Josephson junctions, each junction involving a superconducting $\operatorname{dot}(\mathbf{a}$ or $\mathbf{b})$ and the bulk superconductor $\mathcal{S}$. The Josephson coupling associated with each junction has been computed in Ref. [35] starting from a microscopic, single electron hopping Hamiltonian. Surprisingly, these Josephson couplings can be reinforced by the Coulomb blockade effects in the superconducting dots. In practice, such resonant conditions can be achieved because the individual levels of dots 1, $\mathbf{3}$ (equal by our choice of a symmetric cell) and dot $\mathbf{2}$ can be independently chosen, provided they stay within the superconducting gaps of superconducting dots $\mathbf{a}, \mathbf{b}$. 
In Appendix A, a perturbative argument is provided to show that direct transitions of electrons from $\mathbf{1}$ to $\mathbf{3}$ via $\mathbf{2}$ can be strongly reduced compared to the teleportation process. The amplitude of cotunneling being comparable to that of Andreev transitions [25], the way to suppress cotunneling is by raising the level in $\mathbf{2}$ with respect to $\mathbf{1 , 3}$ (Fig. 2b). Then, contrarily to the teleportation process, cotunneling involves only non-resonant transitions and can be safely neglected to lowest order. We also show show in Appendix A under which conditions the Josephson process can be neglected.

Gate voltages on the five dots of the cell can be tuned such that all the singlet pair transitions in the TP cell are either resonant or blocked by Coulomb interactions (at $T=0$ and neglecting cotunneling). First, pair transitions between dot $\mathbf{a}(\mathbf{b})$ and circuit $\mathbf{S}$ have amplitude $T_{J}^{a, b}$. Second, Andreev pair transitions between dot $\mathbf{a}$ and the pair of dots $(\mathbf{1}, \mathbf{2})$, and between dot $\mathbf{b}$ and the pair of dots $(\mathbf{2}, \mathbf{3})$, have amplitudes $T_{A}^{a, b}$. The resonant condition for these amplitudes will be explicit when writing the coherence terms of the Bloch equations for the reduced density matrix elements. On the opposite, Cooper pair transitions from $\mathbf{a}$ or $\mathbf{b}$ to one of individual dots $\mathbf{1 , 2 , 3}$ are strongly suppressed by Coulomb repulsion. These last assumptions were also considered in 12, 13], where the system formed by a superconductor and two normal dots was shown to behave as a source of entangled Cooper pairs. Non-local (or crossed) Andreev process remains unchanged if the bulk superconductor is a small superconducting island with sizeable Coulomb energy, especially in the resonant case.

We further stress that here the driving force for teleportation is the voltage bias applied to the teleportation cell. The resulting Cooper pair current flowing in the superconducting branch is not a Josephson current, but is instead an Andreev current dragged by the spin-polarized current flowing under the effect of the bias $V$.

\section{Effective Hamiltonian}

¿From what precedes one can derive an effective tunnel Hamiltonian, which involves only pair tunneling within the TP cell and single-electron tunneling to and from the normal reservoirs,

$$
H_{T}^{e f f}=H_{L 1}+H_{R 3}+H_{P}
$$

with

$$
H_{P}=T_{J}\left(\Psi_{a}^{\dagger}+\Psi_{b}^{\dagger}\right) \Psi_{S}+T_{A}^{a} \Psi_{12}^{\dagger} \Psi_{a}+T_{A}^{b} \Psi_{23}^{\dagger} \Psi_{b}+\text { H.c. }
$$

where the $\Psi_{i j}$ destroys a singlet pair in two N-dots and $\Psi_{a, b, S}$ destroys a Cooper pair in the superconducting elements. This Hamiltonian will be used in Section V to derive the average current through the cell.

\section{ELECTROSTATICS OF THE TELEPORTATION CELL}

Let us now discuss the central issue of the Coulomb energy balance in the TP cell. The external variables are the bias voltage $V$ applied between $\mathbf{L}$ and $\mathbf{R}$ and the gate voltages $V_{g \mu}$ applied to dots $\mu=1,2,3, a, b$. For the sake of simplicity, the TP cell is assumed to be symmetric, which means : i) the equality of the tunneling matrix elements between dot pairs a1(2), $\mathbf{b 2}(\mathbf{3})$ and ii) the equality of the capacitances $C_{1 a}=C_{2 a}=C_{2 b}=C_{3 b}=C, C_{a S}=C_{b S}=C_{s}$. Notice that the tunneling amplitudes from reservoir $\mathbf{L}$ or to reservoir $\mathbf{R}$ can instead be different: the symmetry of the device is only assumed within the teleportation cell. One assumes in addition that $C_{L 1}=C_{R 3}=C_{r}$, and that the gate capacitances are all equal to $C_{g}$, which is taken much smaller than $C, C_{s}, C_{r}$. In this section's applications, the teleportation regime will be studied under the assumption $C=C_{r}=C_{s}$ in order to reduce the number of parameters. At temperatures $k_{B} T<<|V|$, the direction of the current through the cell is determined by the sign of $V$, from left $(\mathbf{L})$ to right $(\mathbf{R})$ as a convention. The dot occupation numbers $N_{\mu}$ are defined by the excess charge numbers $n_{\mu}=N_{\mu}-N_{\mu}^{0}$ with respect to a reference state with even occupancies. External voltages can be tuned such as the relevant numbers are $n_{\mu}=0,1$ for $\mathrm{N}$-dots, and $n_{\mu}=0,2$ for S-dots, apart from intermediate quasiparticle states involved in pair transitions, which involve odd charge states. This defines the cell configurations $\left(n_{1} n_{a} n_{2} n_{b} n_{3}\right)$. Incorporating the charge numbers $N_{\mu}^{0}$ into the definition of the effective charges $Q_{\mu}$, one writes the total electrostatic energy of the cell

$$
\left.E_{n_{1} n_{a} n_{2} n_{b} n_{3}}=\frac{1}{2} \sum_{\mu, \nu \in d o t} C_{\mu \nu}^{-1}\left(n_{\mu} e-Q_{\mu}\right)\left(n_{\nu} e-Q_{\nu}\right)\right)-\frac{\left(N_{L}+N_{R}\right) e V}{2}
$$


where $Q_{\mu}=C_{g} V_{g \mu}(\mu=\mathbf{a}, \mathbf{2}, \mathbf{b})$ and $Q_{1}=C_{g} V_{g 1}+C_{r} \frac{V}{2}, Q_{3}=C_{g} V_{g 3}-C_{r} \frac{V}{2} . N_{L}$ and $N_{R}$ are the total number of charges that have passed through junctions $\mathbf{L} / \mathbf{1}$ and $\mathbf{3} / \mathbf{R}$. The voltage drops at these junctions are equal to $V / 2$ due to the symmetry in the junction capacitances.

The electronic transitions within or out of the cell involve total Coulomb energy differences $\Delta E_{i}^{f}=$ $\Delta E_{\left(n_{1} n_{a} n_{2} n_{b} n_{3}\right)_{i}}^{\left(n_{1} n_{a} n_{2} n_{n} n_{3}\right.}+\Delta \varepsilon_{\mu}$ between initial and final states. The second term accounts for the discreteness of the normal dot spectrum, and will be neglected compared to the main Coulomb contribution. Among all possible transitions in the system, several processes can result in a current from reservoir $\mathbf{L}$ to reservoir $\mathbf{R}$. Some of them involve teleportation of the spin state present in $\mathbf{1}$, others not. Selection of the former processes can be achieved at low enough temperature if their energy balance is negative, while that of unwanted processes is positive. Also, spurious processes leading to spin exchange with the reservoirs can be suppressed in this way.

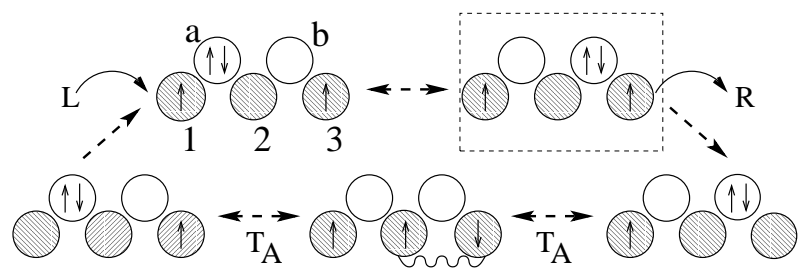

FIG. 4. TP cycle, which operates with a DC bias. "Horizontal" transitions only are resonant. Starting from the framed configuration (upper right), an electron in $\mathbf{3}$ escapes in $\mathbf{R}$; next, a pair (from $\mathbf{b}$ ) creates an entangled state $\mathbf{2}$, $\mathbf{3}$ (wiggly line) with rate $T_{A}$, leaving all N-dots filled. A pair 1, $\mathbf{2}$ then escapes in $\mathbf{a}$. The electron in $\mathbf{3}$ acquires the spin state of dot $\mathbf{1}$, as confirmed by the absorption of a singlet state in $\mathbf{a}$ and the subsequent injection of an electron from $\mathbf{L}$.

In all the above, it is crucial to have a finite charging energy in the superconducting dots. Transitions involving dot occupation by many Cooper pairs would instead follow, leading to further spurious processes. This charging energy is necessary to enforce the desired sequence of events.

\section{A. Allowed transitions within the teleportation cycle}

Allowed transitions are represented in Fig. 1. The sequence may (arbitrarily) start with the evacuation of dot $\mathbf{3}$ triggered by a Cooper pair in $\mathbf{b}$ (see framed section). The electron measured in $\mathbf{R}$ corresponds to a previously teleported state. At the same time, the next electron to be teleported is already sitting in dot $\mathbf{1}$. This allows next to deposit a singlet in dots $\mathbf{b}$ and $\mathbf{c}$ and to ultimately perform the Bell projection, coming back to the initial state. Transitions which do not involve reservoirs are taken to be resonant, while the injection and detection steps from/to the reservoirs are taken to be irreversible. First, the conditions for resonance between configurations (10020), (10101) and (02001) can be deduced from Eq. (14), e.g. $\Delta E_{10020}^{10101}=\Delta E_{10101}^{02001}=0$ :

$$
\begin{aligned}
& Q_{1}+5 Q_{2}+4 Q_{3}+2 Q_{a}-7 Q_{b}=-\frac{3}{2} e \\
& 4 Q_{1}+5 Q_{2}+Q_{3}-7 Q_{a}+2 Q_{b}=-\frac{3}{2} e
\end{aligned}
$$

Similarly, the resonance condition between configurations (12001), (10001) and (10021), e. g. $\Delta E_{10001}^{12001}=\Delta E_{10021}^{10001}=$ 0 leads to

$$
\begin{aligned}
& Q_{1}+5 Q_{2}+4 Q_{3}+2 Q_{a}+8 Q_{b}=13 e \\
& 4 Q_{1}+5 Q_{2}+Q_{3}+8 Q_{a}+2 Q_{b}=13 e
\end{aligned}
$$

These equations can be simplified, assuming $Q_{1}=Q_{3}$ and $Q_{a}=Q_{b}$, which yields

$$
Q_{1}+Q_{2}=\frac{2}{3} e, \quad Q_{a}=\frac{29}{30} e
$$

Notice that the second condition means for S-dots a quasi-resonance between states differing by one Cooper pair. One then finds, as a simple result, that the above resonance conditions for injection or detection processes fix the 
background charge on superconducting dots, and relates those on normal dots. This still leaves one more gate voltage as a free parameter.

Let us turn to the "transport" conditions allowing electronic transitions from $\mathbf{L}$ to $\mathbf{1}$, and from $\mathbf{3}$ to $\mathbf{R}$ to occur (assuming $e V>0$ ). Two cases must be distinguished, depending of the relative energies of the "even" (resonant) states (12001), (10001), (10021) compared to the "odd" (resonant) states (10020), (10101), (02001). First, assume that the even states are more stable (which corresponds to $Q_{1}>\frac{9}{10}$ ). Then the transport conditions can be written as

$$
e \frac{V}{2}>\Delta E_{02001}^{12001}, \Delta E_{10021}^{10020}
$$

which in the present case result in

$$
Q_{1}-\frac{9}{10}<\frac{C V}{e}
$$

On the other hand, in the other case where "odd" states are more stable than "even" ones $\left(Q_{1}<\frac{9}{10}\right)$, one gets similarly

$$
-Q_{1}+\frac{9}{10}<\frac{C V}{e}
$$

The conditions of Eqs (19) and (21) are necessary for the wanted sequence to occur, but still do not prevent spurious transitions from occurring. We now consider all possible processes leaving the sequence states.

\section{B. Unwanted processes}

As discussed earlier, the microscopic Hamiltonian allows for a number of unwanted processes. These can be minimized or reduced for physical reasons, keeping in mind that we can tune the energy levels in the dots.

The first category of unwanted processes concerns the instant at which charges are injected into $\mathbf{1}$ or detected from 3. According to the general TP protocol, a singlet state has to be measured in dots $(\mathbf{1}, \mathbf{2})$, by an irreversible transition to dot a. This means that injection must occur in state (02001), to the exclusion of any other state of the sequence. This is enforced by forbidding the occupation number 2 on dot $\mathbf{1}$, e.g.

$$
e \frac{V}{2}<\Delta E_{12001}^{22001}, \Delta E_{10001}^{20001}, \Delta E_{10021}^{20021}, \Delta E_{10020}^{20020}, \Delta E_{10101}^{20101}
$$

On the other hand, detection from 3 must wait for a classical signal to be sent, that the above measurement has been completed. Since the pair in $\mathbf{a}$, once created, can resonate with $\mathbf{b}$ through $\mathbf{S}$, the signal is nothing but the appearance of a pair in $\mathbf{b}$, which triggers detection from $\mathbf{3}$ by means of the Coulomb repulsion. For this one must avoid spurious transitions from (12001), (10001) and (10101), which implies

$$
e \frac{V}{2}<\Delta E_{12001}^{12000}, \Delta E_{10001}^{10000}, \Delta E_{10101}^{10100}, \Delta E_{02001}^{02000}
$$

The compatibility of Eq. (24) with Eq. (21) requires to satisfy the following conditions

$$
\Delta E_{12001}^{12000}, \Delta E_{10001}^{1000}, \Delta E_{10101}^{10100}, \Delta E_{02001}^{02000}>\Delta E_{10021}^{10020}
$$

These conditions can be shown to be equivalent to the following set of inequalities between inverse capacitances

$$
\begin{aligned}
C_{b 3}^{-1}-C_{a 3}^{-1}>0, & C_{b 3}^{-1}>0 \\
2 C_{b 3}^{-1}-C_{23}^{-1}>0, & 2\left(C_{b 3}^{-1}-C_{a 3}^{-1}\right)+C_{13}^{-1}>0
\end{aligned}
$$

which are always fulfilled, owing to the concavity of the decrease of the inverse capacitance $C_{i j}^{-1}$ with the distance $|i-j|$.

An especially important condition concerns the cotunneling process from dot 1 to dot 2 (see Section II) which involves the possible transitions $(12001) \rightarrow(02101),(10021) \rightarrow(00121),(10001) \rightarrow(00101)$. These transitions involve positive energies provided that $Q_{1}-\frac{8}{15}>0$. A sufficient condition to minimize cotunneling processes is therefore

$$
Q_{1}-\frac{8}{15}>>\gamma_{a}
$$


Other forbidden transitions concern injection from reservoir $\mathbf{R}$, and detection to reservoir $\mathbf{L}$, processes which would contribute to an "inverse" current flowing from $\mathbf{R}$ to $\mathbf{L}$. One can check that this involves the condition

$$
\frac{11}{10}-Q_{1}>\frac{C V}{e} \quad Q_{1}-\frac{1}{6}>\frac{C V}{e}
$$

One could also worry about a process transferring an electron from $\mathbf{1}$ to $\mathbf{3}$ accompanied by a net Cooper pair transfer from $\mathbf{b}$ to $\mathbf{a}$, but the latter happening in reverse order: the absorption of the singlet in a would occur before the creation of the entangled pair in b. Its sequence is $(10020) \rightarrow(02 \overline{1} 20) \rightarrow(02001)$, e. g. the absorption of the singlet in a occurs before creation of the entangled pair in $\mathbf{b}$. This process is however unlikely due to the charge state -1 in dot 2, which renders the crossed Andreev transition non-resonant.

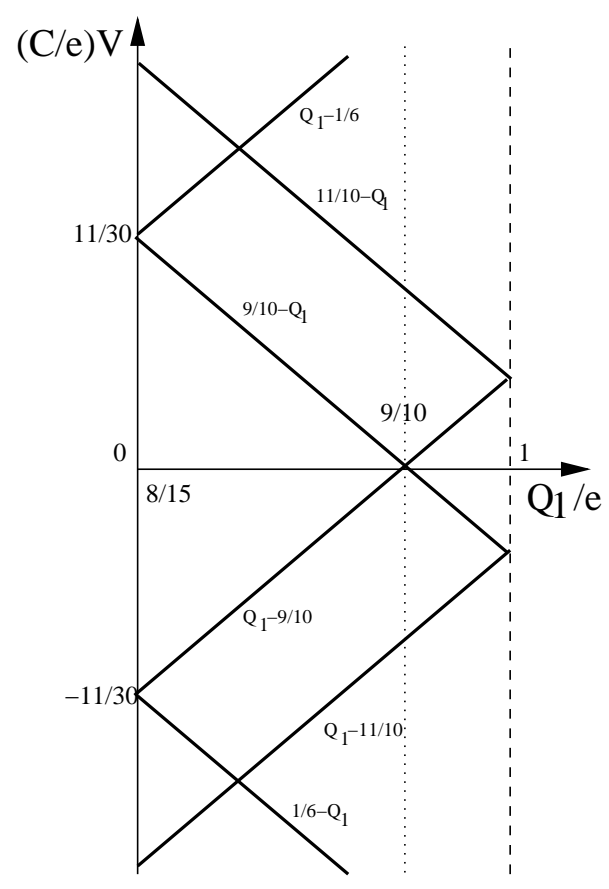

FIG. 5. Stability diagram for the teleportation cycle. The two tilted rectangles indicate the parameter domain for the voltage bias and for the background charge of dot $\mathbf{1}$ which are required to stay in the teleportation cycle.

The operating conditions discussed above can be summarized in a stability diagram (Fig. 5), plotted as a function of $Q_{1}$ and $V$. The two symmetric rectangles tilted at $\pm \pi / 4$ contain the zero temperature working regimes. Outside the vertical lines on the left and on the right, cotunneling is favored. This diagram shows that all the conditions meet in a relatively large portion of the parameter range. Remarkably enough, apart from the "even-odd" degeneracy point at $Q_{1} / e=9 / 10$, there is a threshold voltage for teleportation. This means than the system does no work in the linear regime but instead there is a "Coulomb gap" equal to the energy difference between the even and the odd states participating to the TP sequence. Notice that $V$ is also bounded from above, to avoid spurious transitions during the sequence. The size of the working region guarantees that the system can work in presence of weak thermal and quantum fluctuations. Indeed, the relevant energy scale is a sizeable fraction of the Coulomb charging energy of the dots.

Note that the simplification which consists of choosing the junction capacitances to be equal is by no way restrictive. It can be shown that all the above results hold in general, the only condition being on the relative values of the capacitances $C_{s}$ and $C_{r}$.

\section{CALCULATION OF THE TELEPORTATION CURRENT}

This Section presents the dynamics of the five-dot teleportation cell, described in the above working regime, at zero temperature. The method is that of the master equation describing the dynamics of the reduced density matrix $\left(\sigma_{\mu \nu}\right)$ where the $\mu$ 's are the configuration states retained in the sequence. A microscopic derivation of the master equation for a system of dots, superconductors and normal reservoirs starting from single electron hoppings is presented elsewhere 
[36]. Diagonal elements describe the occupation probabilities of the configurations and non-diagonal ones describe the coherences between them. The latter naturally occur because of the resonant Andreev processes occurring in the TP sequence, and lead to a system of Bloch-like equations [37]. The derivation is inspired by the work of Gurvitz [38, 39] who treated the cases of single and double-dot systems. The generalization starts from an effective Hamiltonian, which is the sum of the Andreev pair Hamiltonian and the one-electron injection/detection terms as in Eq. (12).

Starting with a given initial condition specifying a point of the sequence, for instance the state (10021), with a spin $\sigma$ state in dot 3, the Schrödinger equation is written for the state at instant $t$

$$
|\Psi(t)\rangle=\sum_{\mu, n} b_{\mu, n}(t)|\mu, n\rangle
$$

where the index $n$ contains the information on the quasiparticles which have been transferred (with wave vectors $\left.k_{L}, k_{R}\right)$ from reservoir $\mathbf{L}$ to reservoir $\mathbf{R}$. The reduced density matrix $\left(\sigma_{\mu \nu}\right)$ involves a trace of all transition operators $|\mu\rangle\langle\nu|$ on quasiparticle indices $n$ [38]. The index $\mu=b, 1,3, A, 0, s, t$ runs on the states of the sequence, denoted for simplicity as $|b\rangle=|10021\rangle,|1\rangle=|10020\rangle,|3\rangle=|02001\rangle,|a\rangle=|12001\rangle,|0\rangle=|10001\rangle$, and $|s\rangle,|t\rangle$ involved in the configuration (10101), produced from (10020) as the state $|\sigma\rangle_{1}\left|\psi^{S}\right\rangle_{23}$. The decomposition expressed in Eq. (3) leads to the singlet state $|s\rangle=\left|\psi^{S}\right\rangle_{12}|\sigma\rangle_{3}$ and the triplet combination $|t\rangle=(1 / \sqrt{3}) \sum_{0,+,-}\left|\psi^{T 0,+,-}\right\rangle_{12}\left|\tilde{\sigma}_{0,+,-}\right\rangle_{3}$. The states $\left|\tilde{\sigma}_{0,+,-}\right\rangle_{3}$ are obvious notations for the rotated states appearing in equation (3). Notice that state $|t\rangle$ does not connect to any other state than $|1\rangle$, while $|s\rangle$ connects to $|1\rangle$ and $|3\rangle$.

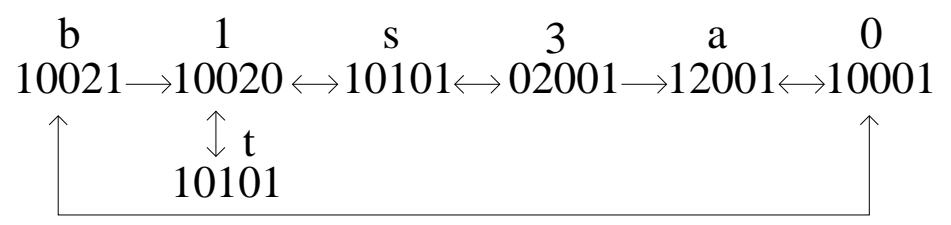

FIG. 6. Labeling of the states which enter the Bloch equations. Their respective connection is identified by arrows. Single arrows represent irreversible processes which involve the transfer of an electron to/from the reservoir.

Let us stress again that spin is perfectly conserved within the teleportation process. Therefore, a general set of closed Bloch equations can be obtained irrespective of the spin direction along an arbitrary axis. This of course holds only in absence of any spin relaxation or decoherence. The spin index is thus omitted in the $\sigma_{\mu \nu}$ :

$$
\begin{aligned}
& \dot{\sigma}_{\mu \mu}=i \sum_{\nu} \Omega_{\mu \nu}\left(\sigma_{\mu \nu}-\sigma_{\nu \mu}\right)-\sum_{\lambda}\left(\Gamma_{\mu \lambda} \sigma_{\mu \mu}-\Gamma_{\lambda \mu} \sigma_{\lambda \lambda}\right) \\
& \dot{\sigma}_{\mu \nu}=i\left(E_{\mu}-E_{\nu}\right) \sigma_{\mu \nu}+i \sum_{\lambda}\left(\sigma_{\mu \lambda} \Omega_{\nu \lambda}-\sigma_{\lambda \nu} \Omega_{\mu \lambda}\right)-\frac{\sigma_{\mu \nu}}{2} \sum_{\lambda}\left(\Gamma_{\mu \lambda}+\Gamma_{\nu \lambda}\right)
\end{aligned}
$$

with $\mu, \nu=a, b, 0,1,3, s, t$, and imaginary coefficients $\left(\Omega_{\mu \nu}\right)$ on the right hand side identify coherent processes, while injection/detection process have real coefficients $\left(\Gamma_{\mu \nu}\right)$. For the teleportation cell, according to Fig. 6 we have $\Omega_{a 0}=\Omega_{0 a}=\Omega_{b 0}=\Omega_{0 b}=T_{J}$, the tunneling rate for Cooper pairs from a to $S$ ( $S$ to b). $\Omega_{1 s}=\Omega_{s 1}=-T_{A} / 2$, $\Omega_{1 t}=\Omega_{t 1}=-\sqrt{3} T_{A} / 2, \Omega_{3 s}=\Omega_{s 3}=T_{A}$ are the Andreev tunneling rates, $\Gamma_{b 1}=\Gamma_{R}, \Gamma_{3 a}=\Gamma_{L}$, all the other $\Omega_{\mu \nu}$ 's and $\Gamma_{\mu \nu}$ 's are zero. The energies $E_{\mu}-E_{\nu}$ of the Cooper pair transitions are included for sake of generality, and are set to zero in the resonant regime. Note that the Andreev amplitudes $T_{A}$ are computed the lowest order in the Appendix, yet here these amplitudes are considered non-perturbative, including all possible round trips between the normal and the superconducting dot. It is therefore possible to consider the limit of a "good" Andreev contact in what follows.

The full system of Bloch equations involve the populations of the 7 above states, and the coherences between states $1,3, s, t$ on one hand, and between states $a, b, 0$ on the other hand. All the other coherences are zero since the corresponding states are coupled by relaxation terms, according to the usual approximation [37]. The Bloch equations are :

$$
\begin{aligned}
\dot{\sigma}_{11} & =-i \frac{1}{2} T_{A}\left(\sigma_{1 s}-\sigma_{s 1}\right)-i \frac{\sqrt{3}}{2} T_{A}\left(\sigma_{1 t}-\sigma_{t 1}\right)+\Gamma_{R} \sigma_{b b} \\
\dot{\sigma}_{s s} & =-i \frac{1}{2} T_{A}\left(\sigma_{s 1}-\sigma_{1 s}\right)+i T_{A}\left(\sigma_{s 3}-\sigma_{3 s}\right) \\
\dot{\sigma}_{t t} & =-i \frac{\sqrt{3}}{2} T_{A}\left(\sigma_{t 1}-\sigma_{1 t}\right)
\end{aligned}
$$




$$
\begin{aligned}
& \dot{\sigma}_{33}=i T_{A}\left(\sigma_{3 s}-\sigma_{s 3}\right)-\Gamma_{L} \sigma_{33} \\
& \dot{\sigma}_{a a}=i T_{s}\left(\sigma_{a 0}-\sigma_{0 a}\right)+\Gamma_{L} \sigma_{33} \\
& \dot{\sigma}_{00}=i T_{s}\left(\sigma_{0 a}-\sigma_{a 0}\right)+i T_{s}\left(\sigma_{0 b}-\sigma_{b 0}\right) \\
& \dot{\sigma}_{b b}=i T_{s}\left(\sigma_{b 0}-\sigma_{0 b}\right)-\Gamma_{R} \sigma_{b b} \\
& \dot{\sigma}_{1 s}=i\left(E_{1}-E_{s}\right)+i\left(-\frac{1}{2} T_{A} \sigma_{11}+T_{A} \sigma_{13}+\frac{1}{2} T_{A} \sigma_{s s}+\frac{\sqrt{3}}{2} T_{A} \sigma_{t s}\right) \\
& \dot{\sigma}_{1 t}=i\left(E_{1}-E_{t}\right)+i\left(-\frac{\sqrt{3}}{2} T_{A} \sigma_{11}+\frac{1}{2} T_{A} \sigma_{s t}+\frac{\sqrt{3}}{2} T_{A} \sigma_{t t}\right) \\
& \dot{\sigma}_{13}=i\left(E_{1}-E_{3}\right)+i\left(T_{A} \sigma_{1 s}+\frac{1}{2} T_{A} \sigma_{s 3}+\frac{\sqrt{3}}{2} T_{A} \sigma_{t 3}\right)-\frac{1}{2} \Gamma_{L} \sigma_{13} \\
& \dot{\sigma}_{3 s}=i\left(E_{3}-E_{s}\right)+i\left(-\frac{1}{2} T_{A} \sigma_{31}+T_{A} \sigma_{33}-T_{A} \sigma_{s s}\right)-\frac{1}{2} \Gamma_{L} \sigma_{3 s} \\
& \dot{\sigma}_{3 t}=i\left(E_{3}-E_{t}\right)+i\left(-\frac{\sqrt{3}}{2} T_{A} \sigma_{31}-T_{A} \sigma_{s t}\right)-\frac{1}{2} \Gamma_{L} \sigma_{3 t} \\
& \dot{\sigma}_{s t}=i\left(E_{s}-E_{t}\right)+i\left(-\frac{\sqrt{3}}{2} T_{A} \sigma_{s 1}+\frac{1}{2} T_{A} \sigma_{1 t}-T_{A} \sigma_{3 t}\right) \\
& \dot{\sigma}_{a 0}=i\left(E_{a}-E_{0}\right)+i T_{J}\left(\sigma_{a b}+\sigma_{a a}-\sigma_{00}\right) \\
& \dot{\sigma}_{b 0}=i\left(E_{b}-E_{0}\right)+i T_{J}\left(\sigma_{b a}+\sigma_{b b}-\sigma_{00}\right)-\frac{1}{2} \Gamma_{R} \sigma_{b 0} \\
& \dot{\sigma}_{a b}=i\left(E_{a}-E_{b}\right)+i T_{J}\left(\sigma_{a 0}-\sigma_{0 b}\right)-\frac{1}{2} \Gamma_{R} \sigma_{a b}
\end{aligned}
$$

which have to be solved, obviously verifying the normalization constraint for the probabilities

$$
\sigma_{11}+\sigma_{s s}+\sigma_{t t}+\sigma_{33}+\sigma_{a a}+\sigma_{00}+\sigma_{b b}=1
$$

In the above system the equations for $\sigma_{\nu \mu}=\sigma_{\mu \nu}^{*}$ are omitted. The stationary solution gives the average current flowing from $\mathbf{L}$ to $\mathbf{1}$ or from $\mathbf{3}$ to $\mathbf{R}$ for each spin direction. This current, denoted as the teleportation current, is given by

$$
I_{t e l, \sigma}=e \Gamma_{L} \sigma_{33}^{\text {stat }}
$$

with $\sigma_{\mu \nu}^{\text {stat }}$ the stationary density matrix elements. Let us first consider the case of resonant Cooper pair transitions. After a straightforward calculation, one obtains quite a simple result

$$
I_{t e l, \sigma}=e \frac{\Gamma_{L} \Gamma_{R}}{\alpha \Gamma_{L}+4 \Gamma_{R}} \frac{T_{A}^{2}}{T_{A}^{2}+\frac{2 \Gamma_{L}^{2} \Gamma_{R}}{\alpha \Gamma_{L}+4 \Gamma_{R}}}
$$

with

$$
\alpha=\frac{3}{2}+\frac{1}{2} \frac{\Gamma_{R}^{2}}{T_{J}^{2}}
$$

One can show that if by chance the transitions from configuration (10020) to (10101) and from (10101) to (02001) are not exactly resonant, the above result still holds provided (10020) and (02001) have the same energy. $T_{A}$ will then be decreased and can be calculated using the lowest order perturbation estimate of the Appendix: for instance, an energy denominator $\left(\Delta E_{10020}^{10101}\right)^{-1}$ will reflect the suppression of the Andreev process describing the singlet injection in $\mathbf{2}, \mathbf{3}$. This situation can be enforced in practice if dots $\mathbf{1}$ and $\mathbf{3}$ on one hand, and dots $\mathbf{a}$ and $\mathbf{b}$ on the other hand, are coupled to the same electrostatic gate in order to preserve the symmetry.

Eqs. (32) to (46) allow to explore the effect of another detuning effect such as the energy difference $\delta$ between (10020) and (02001). One can show that it has a negligible effect provided that $\delta<<\gamma_{L, R}$.

Equating to zero the term $\dot{\sigma}_{b b}$ in Eq. (37) one easily deduces the Cooper pair current from dot a to dot $\mathbf{b}$ :

$$
I_{P}=2 e i T_{J}\left(\sigma_{b 0}^{s t a t}-\sigma_{0 b}^{s t a t}\right)=2 e \Gamma_{R} \sigma_{b b}^{s t a t}=2 e \Gamma_{L} \sigma_{33}^{s t a t}
$$

One thus finds that $I_{P}=2 I_{t e l, \sigma}$. This relationship expresses a very fundamental property : each time a spin state is teleported from $\mathbf{L}$ to $\mathbf{R}$, a Cooper pair passes from dot a to dot $\mathbf{b}$. This is because the pair transfer in the $\mathrm{S}$ 
circuit conditions detection. While in the present work this property can be attributed to the specific model which we have chosen for the teleportation cell, and to the parameters which select the relevant states of the TP cell, the experimental observation of both currents in such a device, on the time scale of the teleportation cycles, would offer a non ambiguous proof of teleportation, as in Ref. [6]. Instead, here the equality is demonstrated only on the average. But it is clear from the operation of the TP sequence, and from the time-dependent solution of Bloch equations, that it is true at the time scale of an elementary spin transfer. This locking of both currents reflects the basic property of TP : splitting a qubit transfer (electron with its spin) into a spin transfer (from $\mathbf{1}$ to $\mathbf{3}$ ) and a classical information transfer from $\mathbf{a}$ to $\mathbf{b}$, which is here nothing but the charge $2 e$. This behaves classically owing to the irreversibility of the transition, driven by the bias $V$. Notice that the classical signal is sent automatically and needs no operation external to the circuit.

Here the inter-dot charging energy plays a crucial role because it conditions the precise sequence. Successive TP cycles follow each other in a sequential way, overlapping the injection step of cycle $N+1$ and detection step of cycle $N$ (Fig. 1). In fact, cycle $N+1$ begins with the injection in $\mathbf{1}$ of an arbitrary spin state. This forces the Bell measurement of the singlet in a for cycle $N$, followed by the classical signal and detection of the $N^{t h}$ teleported spin state in $\mathbf{R}$. Then $\mathbf{b}$ produces the entangled pair for cycle $N+1$, which is measured in a when the spin state for cycle $N+2$ enters in $\mathbf{1}$, and so on.

Let us insist on the irreversibility of both pair production and measurement. Although both processes occur between state of identical energies, the quantum resonance between (10020), (10101) and (02001) ("odd" states) "begins" when the previous electron leaves 3 and "ends" when the next one enters $\mathbf{1}$. Then the resonance between (12001), (10001) and (10021) ("even" states) "begins" and "ends" when the electron leaves $\mathbf{3}$. Change of odd to even states genercally involves a large energy change, of a fraction of $e^{2} / C$ (see Fig. 5). Thus the phase coherence is lost at these transitions, allowing true transfer of classical information. Yet, the spin coherence is preserved during each TP cycle.

One sees that the above protocol has the virtue to work in an automatic way, without any external intervention. It has the great advantage that its speed is only limited by the Andreev amplitude $T_{A}$ and the couplings $\Gamma_{L, R}$, as shown by the average TP current of Eq. (49). It seems plausible that $T_{A}$ can be optimized by reducing the dimensionality of the superconducting dots, as the geometrical factor is known to have a reduction effect in three and two dimensions only. An estimate of currents in the pA range still leads to about $10^{7} \mathrm{TP}$ events per second, a quite sizable quantity. One drawback of such a high cycling value is the difficulty of a time-resolved diagnosis. This will be discussed in a next Section.

\section{COMPARISON WITH THE QUANTUM OPTICS IMPLEMENTATION}

In this work, a primary teleportation diagnosis lies in the nonlocal transfer of the injected electron spin from $L$ into $R$, and in the perfect locking of the average TP current which flows between $L$ and $R$ with the average pair current in the S-circuit. This relies on the assumption - justified by perturbation theory estimates - that processes such as successive cotunneling via the two superconducting dots or such as Josephson tunneling through the central dot, which may affect the fidelity of teleportation, can be neglected: the teleportation channel is then the dominant one.

However, even if one measures average currents (injection and detection current, together with the pair current) and if one finds that these are correlated, this does not constitute a rigorous proof that we are dealing with teleportation. In fact, accidental fluctuations in the gate voltages for instance may pollute the TP process. To be more precise, the true fingerprint of TP is that each time an electron appears in $\mathrm{R}$ with the same spin that was injected from $L$, a Cooper pair passes almost simultaneously from a to b. Quantum mechanics described by our microscopic and effective Hamiltonian confirms explicitly this perfect correlation of electron and Cooper pair currents at the single particle/single Cooper pair level.

In similar situations, encountered experimentally first in quantum optics, such as Bell inequality tests 40,41, a diagnosis which measures correlations between particles independently of the chosen (classical or quantum) description of the apparatus is necessary. Such tests of non-locality allow to rule out a description at the classical level once the outcome of a specific measurement in known.

In the optics experiment [6], a coincidence measurement is performed to isolate the teleportation event. First, two pairs of photons are created at a close time interval with the same laser pulse. The first pair $\mathbf{2 , 3}$ is the entangled pair which is shared by Alice and Bob and which is used to build the three-particle state in the Bell state decomposition. One of the photons of the second pair 1,4 (the test photon $\mathbf{1}$ ) contains the state to be teleported. The other photon of this same pair (4) serves as a simple trigger to signal when the propagation of the photon $\mathbf{1}$ has started. A four-fold coincidence measurement in the trigger detector, in the two detectors needed to signal the measurement of a singlet pair $\mathbf{1}$ and $\mathbf{2}$, and in the detector of the outgoing photon $\mathbf{3}$ allows to confirm the signature of teleportation. 
In nano-circuits, counting single electrons or single Cooper pairs one by one in a transport experiment still represents a challenging task. In the present case, they could in principle be achieved by time resolved capacitive coupling measurements at the injection and detection location, and on one junction of the superconducting circuit. Which quantity needs to be measured to confirm the signature of teleportation? Recall that for the theoretical description of the measurement of entangled states injected from superconductors, Bell inequality tests can be envisioned for situations where a stationary current flows from the superconductors to the detectors, both in a scattering approach [16] and in sequential tunneling scenarios. In the former case, equal time number of particle correlators can be converted into current-current (noise) cross-correlations.

In the present teleportation scenario, assuming the injected particle has a definite spin state, a measurement on both the Cooper pair and on the detected particle is needed. This involves the knowledge of the quantity $\left\langle N_{b}(t)\left(\sigma_{z}\right)_{R}\left(t^{\prime}\right)\right\rangle$, where $N_{b}(t)$ is the excess Cooper pair arriving in $\mathbf{b}$ and $\left(\sigma_{z}\right)_{R}\left(t^{\prime}\right)$ is the electron spin subsequently measured in the detection reservoir $R$ (using a ferromagnetic reservoir). Note that here $t>t^{\prime}$ need to belong to the same cycle. ¿From the teleportation current result of Eq. (49), assuming that the Andreev tunneling amplitude $T_{A}$ is comparable to the rates $\Gamma_{L, R}$, on can estimate the period of each cycle and the condition on the two times above reads $\left(\alpha \Gamma_{L}+\Gamma_{R}\right) / \Gamma_{L} \Gamma_{R}>t-t^{\prime}$. As in the Bell inequality test for solid state devices, the above number correlator can readily be expressed in terms of noise or current-current correlators at finite frequency: $\int d \omega e^{i \omega t}\left\langle I_{P}(t) I_{t e l}(0)\right\rangle$, where $I_{t e l}(t)$ is measured using a spin-polarized reservoir. Note that the choice of having weak injection and escape rates is fully consistent with the working assumptions for using a Bloch equation description.

An experimental test of the device would require to monitor the electron current at the point of injection and detection, and the Cooper pair current between $a$ and $S$ (or $S$ and $b$ ), and to resolve the time correlations [22] between these two currents (in Fig. 2a, such detectors $D_{L, R, S}$ are sketched).

\section{CONCLUSION}

To summarize, an electron spin teleportation scheme which employs a normal/superconducting hybrid nano-device for electrons has been proposed. Although the overall system contains several elements of controlled size and nature (superconducting/normal metal/ferromagnetic) which could prove difficult to integrate, it relies fully on current nanofabrication techniques. The main message of this paper is that teleportation can in principle be achieved in a hybrid superconductor/normal metal nano-device operating without time dependent interactions, in the steady state regime, by simply applying a bias to the device.

We have shown that the teleportation protocol is precisely the same as in the quantum optics experiment, as only the singlet state of the Bell decomposition is used. A novelty however for our system is that the generator for singlet pairs and the detector for such pairs is a superconductor which either breaks Cooper pairs and distributes them in the dots or, alternatively absorbs them, is the same device: the Andreev-dot entangler [13]. We have pointed out that given the microscopic Hamiltonian, several competing processes are possible in this system. These can be minimized by adjusting the electrostatic gates on the dots ands most importantly by working with the condition of resonant pair transition (singlet pairs of electrons in the dots and Cooper pairs in the superconducting elements). This enabled the use of an effective Hamiltonian where the sole single electron jumps consist of the injection and detection processes with the reservoirs. Nonetheless the full operation of the device is not quantum-mechanically coherent, owing to the large energy changes involved in both the injection/measurement and detection processes. Finite capacitances of the dots, together with boundary conditions on the voltage bias and gate voltages, allow to single out a sequence of successive states where teleportation takes place. A Bloch equation description provides the derivation of the teleportation current, which is perfectly locked to the pair current flowing from one superconducting dot to another.

Limiting factors should also be discussed. First, although the transport through the dots is described at the sequential level, it is crucial to maintain spin coherence during the TP sequence (on a time scale $\sim \hbar / \Gamma_{R, L}$, which turns out to be "short" in practical situations). This coherence can be destroyed by spin-orbit coupling, or by collisions with the other electrons within the dot. Such spin-flip processes can be minimized by carefully monitoring the parity of the occupation number of small enough normal dots. Second, the present scheme requires a sufficient amplitude $T_{A}$. This amplitude is reduced by a geometrical factor in two and three dimensions when the two N-S tunnel barriers as spaced farther than a few nanometers [12,13,25.

A more detailed version of the dynamics of our teleportation should in principle also include unwanted processes. Such processes lead to states which lie outside the teleportation cycle states, which can be accessed for instance via cotunneling transitions. Cotunneling process would then be included by coherent couplings of the reduced density matrix elements in the Bloch equation approach, and are then likely to reduce the fidelity of teleportation.

How could this device could be implemented experimentally? At the present time, the best control of quantum dots is achieved with semiconductor dots defined by metallic gates. Nevertheless, hybrid, semiconductor/superconductor 
junctions still present technological challenges. Here, reasonably small barriers have to be achieved between the normal dots and the superconductor in order to maximize the Andreev injection/absorption rate. An alternative would be to define the dots with quasi one-dimensional conductors (nanotubes) placed in contact with superconducting elements. Indeed, bent, gated, contacted carbon nanotubes 42 have demonstrated Coulomb blockade behavior. Concerning the contact with a superconductor-nanotube junction, there is some hope that the geometrical constraint which operates in two and three dimensions is relaxed [27,28]. Also, the feasibility of this proposal relies on the control of the single electron injection and detection process. Efficient spin filters $L, R$, are already available at low temperatures 43.

Finally, it is legitimate to ask about the practical range over which the electron spin state can be teleported. In the present situation, using low temperatures, it is likely to be of a few microns. The proposed setup is generalizable to $2 \mathcal{N}+1$ normal dots, together with $\mathcal{N}$ superconducting circuits $(2 \mathcal{N}$ S-dots): TP of a spin state in dot 1 onto dot $2 \mathcal{N}+1$ can be achieved by a swapping process [9], thus extending the range of TP.

\section{ACKNOWLEDGMENTS}

Discussions with V. Bouchiat are gratefully acknowledged. LEPES is under convention with UJF and INPG, Grenoble. One of us (T.M.) wishes to thank NTT Basic Research Laboratories for their hospitality.

\section{APPENDIX A: PERTURBATIVE CALCULATION OF PAIR TUNNELING, COTUNNELING AND JOSEPHSON AMPLITUDES}

In order to justify our assumptions, we present lowest order perturbative estimates of the electron transfer process in the teleportation cell displayed in Figure 3. For simplicity, the expressions below are derived with our working assumption that the charging energies of the dots are much smaller than the superconducting gap $\Delta$ (in $\mathbf{a}, \mathbf{b}, \mathbf{S}$ ). Calculations are performed using the T-matrix approximation, in which the "effective tunneling Hamiltonian" is specified by:

$$
H_{T}^{e f f}=H_{T}+H_{T} \sum_{n}\left[\frac{1}{i \eta+\varepsilon_{i}-H_{0}-H_{C}} H_{T}\right]^{n}
$$

with $\varepsilon_{i}$ the initial energy and the Hamiltonian in the denominator excludes all single electron hoppings. $\eta$ is an infinitesimal. We are interested in events which connect an initial state $|i\rangle$ (specified by the occupation configurations $n_{1}, n_{a}, n_{2}, n_{b}, n_{3}$ of the dots) which is connected by $H_{T}^{e f f}$ to a final state $|f\rangle$ with the same energy.

\section{Second order processes}

Assuming no external phase difference across the superconductors, it is straightforward to derive the effective amplitudes for pair tunneling $\left(T_{A}\right)$, cotunneling $\left(T_{C}\right)$ and Josephson tunneling $\left(T_{J}\right)$ between the superconducting elements, to second order in the single electron hopping amplitudes $t_{\mu \nu} . T_{J}^{a}\left(T_{J}^{b}\right)$ involves an intermediate state with one Bogolubov quasiparticle in $\mathbf{S}$ or $\mathbf{a}(\mathbf{b})$, while $T_{A}^{a}\left(T_{A}^{b}\right)$ involves an intermediate state with one quasiparticle in $\mathbf{a}$ (b). The amplitudes $T_{J}^{a, b}$ have been calculated in Ref. [35] and are not reproduced here. Summing over all possible intermediate states, the crossed Andreev amplitudes are given by:

$$
\begin{aligned}
T_{A}^{a} & \simeq 2 \sum_{k} u_{k}^{a} v_{k}^{a} t_{1 a} t_{2 a} /\left(i \eta-E_{k}^{a}\right) \\
T_{A}^{b} & \simeq 2 \sum_{k} u_{k}^{b} v_{k}^{b} t_{2 b} t_{3 b} /\left(i \eta-E_{k}^{b}\right)
\end{aligned}
$$

Cotunneling amplitudes are specified in a similar way:

$$
\begin{aligned}
T_{C}^{a} & \simeq \sum_{k} t_{1 a} t_{a 2}\left(\left|u_{k}^{a}\right|^{2}-\left|v_{k}^{a}\right|^{2}\right) /\left(i \eta-E_{k}^{a}\right) \\
T_{C}^{b} & \simeq \sum_{k} t_{2 b} t_{b 3}\left(\left|u_{k}^{b}\right|^{2}-\left|v_{k}^{b}\right|^{2}\right) /\left(i \eta-E_{k}^{b}\right)
\end{aligned}
$$


These amplitudes are reduced from an ideal value of order $\gamma_{\alpha}$ by the necessary propagation of the virtual intermediate state quasiparticle between the two junctions, at a distance $l$. This involves a geometrical factor 12, 13, 25, of order $f(l)=\left(\lambda_{S} / l\right)^{2} e^{-l / \pi \xi_{0}}$ in the clean limit ( $\lambda_{S}$ is the Fermi length in the superconductor and $\xi_{0}$ is the coherence length).

Note that both the Andreev and the cotunneling amplitudes of Eqs. (A3) and (A5) contain denominators $\left(i \eta-E_{k}^{a}\right.$ ) which involve the superconducting gap. This, however does not imply that the Amplitudes for such processes are proportional to $\Delta^{-1}$, as one needs to take into account the energy dependence of $u_{k}^{a, b}$ and $v_{k}^{a, b}$ in order to compute the sums. In fact, these processes have either no dependence on the gap - the case of the Andreev process - or a logarithmic dependence $\ln (W / \Delta)$, where $W$ is the bandwidth of the superconductor in the normal state - for the case of cotunneling. Discarding numerical factors of order unity, one finds:

$$
\begin{array}{r}
T_{A}^{a} \sim t_{1 a} t_{2 a} \nu_{S} f(l) \\
T_{A}^{b} \sim t_{2 b} t_{3 b} \nu_{S} f(l) \\
T_{C}^{a} \sim t_{1 a} t_{a 2} \nu_{S} \ln (W / \Delta) f(l) \\
T_{C}^{b} \sim t_{2 b} t_{b 3} \nu_{S} \ln (W / \Delta) f(l)
\end{array}
$$

with $\nu_{S}$ the density of states of the superconductor in the normal state. For typical physical parameters, the logarithm is of order one, so the Andreev and the cotunneling process have a comparable magnitude.

\section{2. fourth order processes}

We consider three types of fourth-order tunneling processes which are relevant to our problem: a) teleportation processes; b) the succession of two cotunneling processes which result in the spin conserving transfer of an electron from 1 to $\mathbf{3}$ (an unwanted process which pollutes teleportation); c) Josephson tunneling from $\mathbf{a}$ to $\mathbf{b}$ via dot 2.

For the teleportation process we start with the state (10020) as in Fig. 11. Transferring one electron on 2 or 3 creates a quasiparticle in b (10110 or 10011), which is subsequently destroyed when the intermediate state with the electrons in the normal dots is reached (10101). The final state (02001) can be reached via either of the two states (11001 or 01101) with one quasiparticle in a each. The off-resonance (OR) teleportation tunneling amplitude then reads:

$$
T_{T e l, O R} \simeq-\left(i \eta-\Delta E_{10020}^{10101}\right)^{-1} \sum_{k} \sum_{q} \frac{4 t_{b 2} t_{b 3} t_{a 1}^{*} t_{a 2}^{*} u_{k}^{b} v_{k}^{b} u_{q}^{a *} v_{q}^{a *}}{E_{k}^{b} E_{q}^{a}}
$$

Note that by specifying that the dots level energies in 1, $\mathbf{2}$ are located at an equal distance but opposite location with respect to the superconducting chemical potential, the resonance condition is enforced: $\Delta E_{10020}^{10101}=0$ (also $\Delta E_{10101}^{02001}=0$ ) and this expression diverges (except for the presence of $i \eta$ ). A more careful analysis would show that an re-summation of all the terms in the perturbation series leads to a finite amplitude in this resonant situation. As specified in the previous section, the resonant regime is the working assumption of our teleportation proposal.

For the successive two cotunneling events, it is possible to write down a general expression for transitions from the state $n_{1} n_{a} n_{2} n_{b} n_{3}$ to the final state $\left(n_{1}-1\right) n_{a} n_{2} n_{b}\left(n_{3}+1\right)$ via the intermediate state $\left(n_{1}-1\right) n_{a}\left(n_{2}+1\right) n_{b} n_{3}$ :

$$
T_{2 C o t} \simeq-\left(\Delta E_{n_{1} n_{a} n_{2} n_{b} n_{3}}^{\left(n_{1}-1\right) n_{a}\left(n_{2}+1\right) n_{b} n_{3}}\right)^{-1} \sum_{k} \sum_{q} \frac{t_{1 a} t_{a 2} t_{2 b} t_{b 3}\left(\left|u_{k}^{a}\right|^{2}-\left|v_{k}^{a}\right|^{2}\right)\left(\left.u_{q}^{b}\right|^{2}-\left|v_{q}^{b}\right|^{2}\right)}{E_{k}^{a} E_{q}^{b}}
$$

Computing the sums over momentum, this is estimated to be:

$$
T_{2 C o t} \sim-\left(\Delta E_{n_{1} n_{a} n_{2} n_{b} n_{3}}^{\left(n_{1}-1\right) n_{a}\left(n_{2}+1\right) n_{b} n_{3}}\right)^{-1} t_{1 a} t_{a 2} t_{2 b} t_{b 3} \nu_{S}^{2} \ln ^{2}(W / \Delta)=\left(\Delta E_{n_{1} n_{a} n_{2} n_{b} n_{3}}^{\left(n_{1}-1\right) n_{a}\left(n_{2}+1\right) n_{b} n_{3}}\right)^{-1} T_{C}^{a} T_{C}^{b}
$$

\section{Estimation of the cotunneling current}

Under the resonance condition, the energy denominator $E_{n_{1} n_{a} n_{2} n_{b} n_{3}}^{\left(n_{1}-1\right) n_{2}\left(n_{2}+1\right) n_{b} n_{3}}$ is "large" and successive cotunneling can be minimized. Indeed, using Eq. (14) and the definition of the capacitance matrix, one obtains:

$$
\Delta E_{n_{1} n_{a} n_{2} n_{b} n_{3}}^{\left(n_{1}-1\right) n_{2}\left(n_{2}+1\right) n_{b} n_{3}} / e^{2}=\frac{1 / 2-n_{1}+Q_{1}}{C+C_{r}+C_{g}}+\frac{1 / 2+n_{2}-Q_{2}}{2 C+C_{g}}-\frac{n_{b}-Q_{b}}{C}
$$


At this point we use our working assumption that all capacitances are equal, together with the resonance condition which fixes $Q_{a}=Q_{b}=29 / 30$. Furthermore we choose a value of $Q_{1}$ which is located well into the stability regions of Fig. 5. For a given initial configuration, such as $n_{1}=1, n_{2}=0=n_{3}, n_{a}=0, n_{b}=2$, as in the bottom right corner of Fig. 4 , one obtains that:

$$
\Delta E_{10020}^{00120} \sim \frac{e^{2}}{C}
$$

with a numerical prefactor of order unity. One can therefore favor processes involving two sequential Andreev processes while at the same time reducing the effect of one-electron processes from $\mathbf{1}$ to $\mathbf{3}$.

Granted, our finding that the intermediate state for the successive cotunneling process has a "large" charging energy $e^{2} / C$ is strictly speaking not sufficient to convince oneself that this process can be ruled out: the typical current associated with this process needs to be compared to the teleportation current of Eq. (49). To estimate this cotunneling current, we consider $T_{2 C o t}$ to be the effective hopping amplitude between 2 dots, dot 1 and dot $\mathbf{3}$, both of which are connected to reservoirs $\mathbf{L}$ and $\mathbf{R}$. A Bloch equation approach was used in Ref. [44] to describe this situation. The corresponding current reads:

$$
I_{2 C o t}=e \frac{\Gamma_{L} \Gamma_{R}}{\Gamma_{L}+\Gamma_{R}} \frac{T_{2 C o t}^{2}}{T_{2 C o t}^{2}+\Gamma_{L} \Gamma_{R} / 4}
$$

Note that the teleportation current and the cotunneling current become comparable when $T_{A}^{2}, T_{2 C o t}^{2}>\Gamma_{L} \Gamma_{R}$. The teleportation cell requires the opposite limit. Indeed, the injection and evacuation from/to the reservoirs need to be efficient enough in order to avoid spurious processes. One is now in a position to justify why cotunneling can be neglected when compared to the teleportation current. Assuming $\Gamma_{L} \sim \Gamma_{R}, \Gamma_{L, R} \gg T_{A}, T_{2 C o t}$ and neglecting numerical factors, the two currents become:

$$
\begin{aligned}
I_{t e l, \sigma} & \sim e \frac{T_{A}^{2}}{\Gamma_{L, R}} \\
I_{2 C o t} & \sim e \frac{T_{2 C o t}^{2}}{\Gamma_{L, R}}
\end{aligned}
$$

So one simply needs to compare $T_{A}$ and $T_{2 C o t}$. Using the estimate of Eq. A11 (assuming all single electron hoppings to be comparable), the condition for neglecting the cotunneling current becomes:

$$
T_{2 C o t} \sim \frac{T_{A}^{2}}{\Delta E_{n_{1} n_{a} n_{2} n_{b} n_{3}}^{\left(n_{1}-1\right) n_{a}\left(n_{2}+1\right) n_{b} n_{3}}} \ll T_{A}
$$

that is, the crossed Andreev reflection hopping is required to be much smaller than the energy difference between the initial and the intermediate state implied by the sequential cotunneling process. We have seen in the above that this energy difference is maximized when the capacitive energy $e^{2} / C$ is large, this is a confirmation of the crucial role played by the capacitive couplings in order to select the proper teleportation sequence.

\section{4. "Other" (less relevant) fourth order processes}

Note that there are other higher order cotunneling processes, for instance those which involve intermediate states with two quasiparticles (one in $\mathbf{a}$ and one in $\mathbf{b}$ at the same time). One argues here that they are much weaker because the corresponding energy denominators all contain the superconducting gap $\Delta^{3}$. A typical contribution would read (this time neglecting the charging energies in front of the gap):

$$
T_{2 C o t}^{\prime} \simeq-\sum_{k} \sum_{q} \frac{t_{1 a} t_{a 2} t_{2 b} t_{b 3}\left(\left|u_{k}^{a}\right|^{2}-\left|v_{k}^{a}\right|^{2}\right)\left(\left.u_{q}^{b}\right|^{2}-\left|v_{q}^{b}\right|^{2}\right)}{E_{k}^{a} E_{q}^{b}\left(E_{k}^{a}+E_{q}^{b}\right)}
$$

The summations over momenta can be performed, for instance, provided that, for instance $\left(E_{k}^{a}+E_{q}^{b}\right)$ is replaced by $E_{k}^{a}$ or $E_{q}^{b}$. This yields:

$$
\left|T_{2 C o t}^{\prime}\right|<\left|\sum_{k} \sum_{q} \frac{t_{1 a} t_{a 2} t_{2 b} t_{b 3}\left(\left|u_{k}^{a}\right|^{2}-\left|v_{k}^{a}\right|^{2}\right)\left(\left.u_{q}^{b}\right|^{2}-\left|v_{q}^{b}\right|^{2}\right)}{\left(E_{k}^{a}\right)^{2} E_{q}^{b}}\right| \sim t_{1 a} t_{a 2} t_{2 b} t_{b 3} \frac{\nu_{S}^{2}}{\Delta} \ln W / \Delta
$$


One therefore sees that the latter contribution is smaller than that of Eq. (A11) by a factor $\Delta E_{n_{1} n_{a} n_{2} n_{b} n_{3}}^{\left(n_{1}-1\right) n_{a}\left(n_{2}+1\right) n_{b} n_{3}} / \Delta$. Recall that throughout this work, charging energies are assumed to be smaller than $\Delta$.

Finally, we evaluate some processes contributing to the Josephson tunneling amplitudes (pair tunneling from $\mathbf{b}$ to $\mathbf{a}$ via the central dot 2). The transport properties of a Josephson junction containing an impurity level (with charging energy) in the tunnel barrier has been detailed in Ref. [34]. Two situations are considered here. First, assume that dot 2 is empty. The transitions involve an initial state $n_{1} 002 n_{3}$ and a final state $n_{1} 200 n_{3}$. This transition necessarily involves (at the beginning and at the end) 2 intermediate states $n_{1} 011 n_{3}$ and $n_{1} 110 n_{3}$ with one quasiparticle into a or $\mathbf{b}$. The third intermediate state then has two possibilities: either it involves a quasiparticle in both $\mathbf{a}$ and $\mathbf{b}$ which gives a tunneling amplitude:

$$
T_{J 2}^{(1)} \approx-\sum_{k} \sum_{q} \frac{t_{b 2}^{2} t_{2 a}^{2} u_{q}^{a} v_{q}^{a} u_{k}^{b *} v_{k}^{b *}}{E_{q}^{a}\left(E_{q}^{a}+E_{k}^{b}\right) E_{k}^{b}} \sim-\frac{t_{b 2}^{2} t_{2 a}^{2} \nu_{S}^{2}}{\Delta}
$$

or it implies double occupancy of the central dot:

$$
T_{J 2}^{(2)} \approx-\left(\Delta E_{n_{1} 002 n_{3}}^{n_{1} 020 n_{3}}\right)^{-1} \sum_{k} \sum_{q} \frac{t_{b 2}^{2} t_{2 a}^{2} u_{q}^{a} v_{q}^{a} u_{k}^{b *} v_{k}^{b *}}{E_{q}^{a} E_{k}^{b}} \sim-\frac{t_{b 2}^{2} t_{2 a}^{2} \nu_{S}^{2}}{\Delta E_{n_{1} 002 n_{3}}^{n_{1} 02 n_{3}}} .
$$

When one imposes a phase difference between $\mathbf{a}$ and $\mathbf{b}$, the fact that the central dot is empty at the initial state makes this junction a "0" junction. On the other hand, if dot $\mathbf{2}$ is initially occupied, we look for transitions such as $n_{1} 012 n_{3} \rightarrow n_{1} 102 n_{3} \rightarrow n_{1} 111 n_{3} \rightarrow n_{1} 201 n_{3} \rightarrow n_{1} 210 n_{3}:$

$$
T_{J 2}^{(3)} \approx \sum_{k} \sum_{q} \frac{t_{b 2}^{2} t_{2 a}^{2} u_{q}^{a} v_{q}^{a} u_{k}^{b *} v_{k}^{b *}}{E_{q}^{a}\left(E_{q}^{a}+E_{k}^{b}\right) E_{k}^{b}} \sim-T_{J 2}^{(1)} .
$$

On the other hand one can have the sequence $n_{1} 012 n_{3} \rightarrow n_{1} 102 n_{3} \rightarrow n_{1} 2(-1) 2 n_{3} \rightarrow n_{1} 201 n_{3} \rightarrow n_{1} 210 n_{3}$ with the amplitude:

$$
T_{J 2}^{(4)} \approx\left(\Delta E_{n_{1} 012 n_{3}}^{n_{1} 2(-1) 2 n_{3}}\right)^{-1} \sum_{k} \sum_{q} \frac{t_{b 2}^{2} t_{2 a}^{2} u_{q}^{a} v_{q}^{a} u_{k}^{b *} v_{k}^{b *}}{E_{q}^{a} E_{k}^{b}} \sim \frac{t_{b 2}^{2} t_{2 a}^{2} \nu_{S}^{2}}{\Delta E_{n_{1} 012 n_{3}}} .
$$

The latter two transitions lead to a $\pi$-junction behavior. Note that all the Josephson processes either involve three powers of the superconducting gap in their denominators, or they are proportional to $\Delta^{-2}$ times the inverse of the Coulomb charging energy for double occupancy of normal dot 2. The choice of normal dots with a small total capacitance therefore allows to neglect these processes when compared to the (resonant) teleportation amplitude.

[1] C. H. Bennett, G. Brassard, C. Crépeau, R. Josza, A. Peres and W. K. Wooters, Phys. Rev. Lett. 70, 1895 (1993).

[2] A. Aspect, J. Dalibard and G. Roger, Phys. Rev. Lett. 49, 1804 (1982)

[3] L. Mandel, Rev. Mod. Phys. 71, S274 (1999); A. Zeilinger, Rev. Mod. Phys. 71, S288 (1999).

[4] A. Einstein, B. Podolsky, and N. Rosen, Phys. Rev. Lett. 47, 777 (1935).

[5] W. K. Wootters and W. H. Zurek, Nature 299, 802 (1982).

[6] D. Bouwmeester, J.-W. Pan, K. Mattle, M. Eibl, H. Weinfurter and A. Zeilinger, Nature (London) 390, 575 (1997).

[7] D. Boschi, S. Branca, F. De Martini, L. Hardy and S. Popescu, Phys. Rev. Lett. 80, 1121 (1998); A. Furusawa, J. Sorensen, S. L. Braunstein, C. Fuchs, H. J. Kimble, and E. S. Polzik, Science 282, 706 (1998); S. L. Braunstein and H. J. Kimble, Phys. Rev. Lett. 80, 869 (1998).

[8] M. A. Nielsen, E. Knill and R. Laflamme, Nature (London) 396, 52 (1998); I. L. Chuang, L. M. K. Vandersypen, XinlanZhou, D. W. Leung and S. Lloyd, Nature 393, 143 (1998); J. A.Jones, M. Mosca and R. H. Hansen, Nature 393, 344 (1998); L. M. K. Vandersypen, M. Steffen, G. Breyta, C. S. Yannoni, R. Cleve and I. L. Chuang, Phys. Rev. Lett. 85, 5452 (2000); L. M. K. Vandersypen, M. Steffen, G. Breyta, C. S. Yannoni, M. H. Sherwood and I. L. Chuang, Nature 414, 883 (2001).

[9] D. Bouwmeester, A. Ekert and A. Zeilinger, The Physics of Quantum Information (Springer-Verlag, Berlin, 2000).

[10] C. H. Bennett, D. P. DiVincenzo, J. A. Smolin and W. K. Wootters, Phys. Rev. A 54, 3824 (1996).

[11] A. A. Larionov, L. E. Fedichkin and K. A. Valiev, Nanotechnology 12, 536 (2001); B. Kane, Phys. Rev. Lett 90, 087901 (2003). 
[12] M. S. Choi, C. Bruder and D. Loss, Phys. Rev. B 62, 13569 (2000).

[13] P. Recher, E. V. Sukhorukov and D. Loss, Phys. Rev. B 63, 165314 (2001).

[14] G. B. Lesovik, T. Martin and G. Blatter, Eur. Phys. J. B 24, 287 (2001).

[15] G. Deutscher and D. Feinberg, Appl. Phys. Lett. 76, 487 (2000).

[16] N. Chtchelkatchev, G. Blatter, G. Lesovik and T. Martin, Phys. Rev. B 66, 161320 (2002).

[17] J. A. Reina and N. F. Johnson, Phys. Rev. A 63, 012303 (2000).

[18] D. Loss and D. P. DiVincenzo Phys. Rev. A 57, 120 (1998).

[19] W. D. Oliver, F. Yamaguchi and Y. Yamamoto, Phys. Rev. Lett. 88, 037901 (2002).

[20] D. S. Saraga and D. Loss, Phys. Rev. Lett. 90, 166803 (2003).

[21] T. Martin and R. Landauer, Phys. Rev. B 45, 1742 (1992); M. Büttiker, ibid. 46, 12485 (1992); Phys. Rev. Lett. 65, 2901 (1990).

[22] M. Henny, S. Oberholzer, C. Strunk, T. Heinzel, K. Ensslin, M. Holland and C. Schönenberger, Science 284, 296 (1999); W. Oliver, J. Kim, R. Liu and Y. Yamamoto, ibid, 299 (1999); T. Martin and R. Landauer, Phys. Rev. B 45, 1742 (1992).

[23] M. H. Devoret and H. Grabert, In Single Charge Tunneling, H. Grabert and M. H. Devoret eds. (Plenum, New York 1992); D. Estève, ibid.

[24] O. Sauret, D. Feinberg and T. Martin, Eur. Phys. J. B 32, 545 (2003)

[25] G. Falci, D. Feinberg and F. W. J. Hekking, Europhys. Lett. 54, 255 (2001).

[26] R. Mélin and D. Feinberg, Eur. Phys. J. B26, 101 (2002).

[27] P. Recher and D. Loss, Phys. Rev. B 65, 165327 (2002).

[28] V. Bouchiat, N. Chtchelkatchev, D. Feinberg, G. Lesovik, T. Martin and J. Torres, Nanotechnology 14, 77 (2003).

[29] For a clean superconductor, $\xi=\xi_{0}$ is the BCS coherence length. In a dirty superconductor, with mean-free path $l<\xi_{0}$, quasiparticle decay on the coherence length $\xi \sim \sqrt{\xi_{0} l}$ and the prefactor varies like $R^{-1}$ instead of $R^{-2}$ (D. Feinberg, ArXiv cond-mat/0307099, N. M. Chtchelkatchev and M. Mar'enko, ArXiv cond-mat/0306552).

[30] D. Bouwmeester, J.-W Pan, H. Weinfurter, and A. Zeilinger, J. Mod. Opt. 47, 279 (2000).

[31] M. Tinkham, Introduction to Superconductivity (2nd ed.) (Mc Graw-Hill, New York, 1996).

[32] C. W. J. Beenakker, Phys. Rev. B 44, 1646 (1991).

[33] D. V. Averin and Yu. V. Nazarov, in Single Charge Tunneling, H. Grabert and M.H. Devoret eds. (Plenum, New York 1992).

[34] L. I. Glazman and K. A. Matveev, Pis'ma Zh. Eksp. Teor. Fiz. 49, 570 (1989) [JETP Lett. 49, 659 (1989)].

[35] K. A. Matveev, M. Gisselfält, L. I. Glazman, M. Jonson and R. I. Shekhter, Phys. Rev. Lett. 70, 2940 (1993).

[36] O. Sauret, D. Feinberg, T. Martin, in preparation.

[37] C. Cohen-Tannoudji, J. Dupont-Roc and G. Grynberg, Atom-Photon Interactions: Basic Processes and Applications (Wiley, New York, 1992).

[38] S. A. Gurvitz and Ya. S. Prager, Phys. Rev. B 53, 15932 (1996).

[39] U. Geigenmüller and G. Schön, Europhys. Lett. 10, 765 (1989); D. V. Averin, A. N. Korotkov and K. K. Likharev, Phys. Rev. B 44, 6199 (1991).

[40] J.S. Bell, Rev. Mod. Phys. 38, 447 (1966); J.F. Clauser, M. A. Horne, A. Shimony and R. A. Holt, Phys. Rev. Lett. 23, 880 (1969).

[41] A. Aspect, J. Dalibard, and G. Roger, Phys. Rev. Lett. 49, 1804 (1982); L. Mandel, Rev. Mod. Phys. 71, S274 (1999); A. Zeilinger, Rev. Mod. Phys. 71, S288 (1999).

[42] A. Bezryadin, A.R.M. Verschueren, S.J. Tans, and C. Dekker, Phys. Rev. Lett. 80, 4036-4039 (1998)

[43] R. Fiederling, M. Keim, G. Reuscher, W. Ossau, G. Schmidt, A. Waag and L. W. Molenkamp, Nature (London) 402, 787 (1999); Y. Ohno, D. K. Young, B. Beschoten, F. Matsukura, H. Ohno and D. D. Awschalom, Nature (London) 402, 790 (1999).

[44] S. A. Gurvitz, Phys. Rev. B 57, 6602 (1998). 